\title{
Der Chor von St. Moritz in Olmütz (Olomouc) im Spannungsfeld architekturgeschichtlicher Erfindungen des mitteleuropäischen Spätmittelaltelters. Alte Beobachtungen und neue Erkenntnisse
}

\author{
Sophie MORAWITZ
}

\begin{abstract}
The reconstruction of St Maurice in Olomouc was one of the most important building projects in late medieval Moravia. Research assumes that the work began in 1415 and continued, with several interruptions, into the late 15 th century. This paper wants to critically examine the dating of the choir as eastern part of the parish church parish church between 1453 and 1483/1492.

It approaches the building enterprise with a detailed analysis of the stylistic, typological and archaeological findings and highlights the dynamics behind the outlined designs. Especially the ambitious and, for this region, unusual designs share far-reaching artistic connections with important centers such as Wrocław, Brno and Vienna. Therefore, this paper proposes that the project's exceptional value is imbedded in Central Europe's architectural history.
\end{abstract}

Keywords: St Moritz Olomouc - Moravia - Staggered Choir - Vienna Mason's Lodge - Late Middle Ages - Inter-Regional Artistic Connections - 15th Century - 14th Century - Brno Mason's Lodge Czech - Austria - Poland - Wroclaw

Das Interesse an der Olmützer Stadtpfarrkirche St. Moritz (Abb. 1-2) ${ }^{1}$ konzentrierte sich in den letzten Jahrzehnten auf die westlichsten und zu-

\footnotetext{
Dieser Artikel beruht auf den Ergebnissen einer 2019 von der Autorin an der Universität Wien eingereichten Masterarbeit zum selben Thema. Dementsprechend können alle hier getätigten Aussagen mit voller Beschreibung, stilistischer sowie typologischer Einordnung und ausführlicher Literaturangabe dieser Arbeit entnommen werden. Dazu siehe: MORAWITZ, S.: Der Chor von St. Moritz, in Olmütz, (Olomouc). Fragen zu dessen Bauchronologie und seinem kunsthistorischen Stellenwert, Masterarbeit (unpubl.), Wien 2019. Für die Unterstützung und Hilfe in der Vorbereitung dieses Aufsatzes (sowie der zugrundeliegenden Arbeit) bedanke ich mich bei: Michael Viktor Schwarz, Tim Juckes, Ondřej Belšík, Hana Myslivečková, Johann Josef Böker, Günther Buchinger, Petra Ptakova, Anna-Marie Kroupová, Jakob Kremsner, Cedric Huss, Nina Harm und Oliver Fries.

1 Da sich die bisher publizierten Baupläne der Moritzkirche auf-
}

gleich jüngsten Ausbauteile (Schlingrippengewölbe, Westportal, Doppelwendeltreppe), was sich aus der ins Auge springenden Raffinesse dieser Schöp-

grund ihrer vereinfachten und idealisierenden Ausführung als unzureichend und wenig aussagekräftig erwiesen, wurde auf Basis eigener Messungen und eines im Jahre 1946 von Alois Štefka angefertigten, und 1973 ergänzten, Vermessungsplanes ein neuer Grundrissplan angefertigt. Dieser entspricht dem tatsächlichen Bestand und bietet somit eine wichtige Grundlage für jegliche Auseinandersetzung mit dem Kirchenbau. Darüber hinaus wurden auch Aufmaße der Wandvorlagen im Chor von St. Moritz und der Wiener Augustinerkirche erstellt. Diese Architekturzeichnungen basieren auf Vermessungen, welche im Falle von Olmütz von Ondřej Belšík und Hana Myslivečková vom NPÚ ÚOP v Olomouci und im Falle von Wien durch Nina Harm und die Verfasserin im Jahre 2020 schematisch aufgenommen wurden. Die technische Umsetzung der eben angegebenen Architekturzeichnungen erfolgte durch Jakob Kremsner. Der Plan von Alois Štefka 


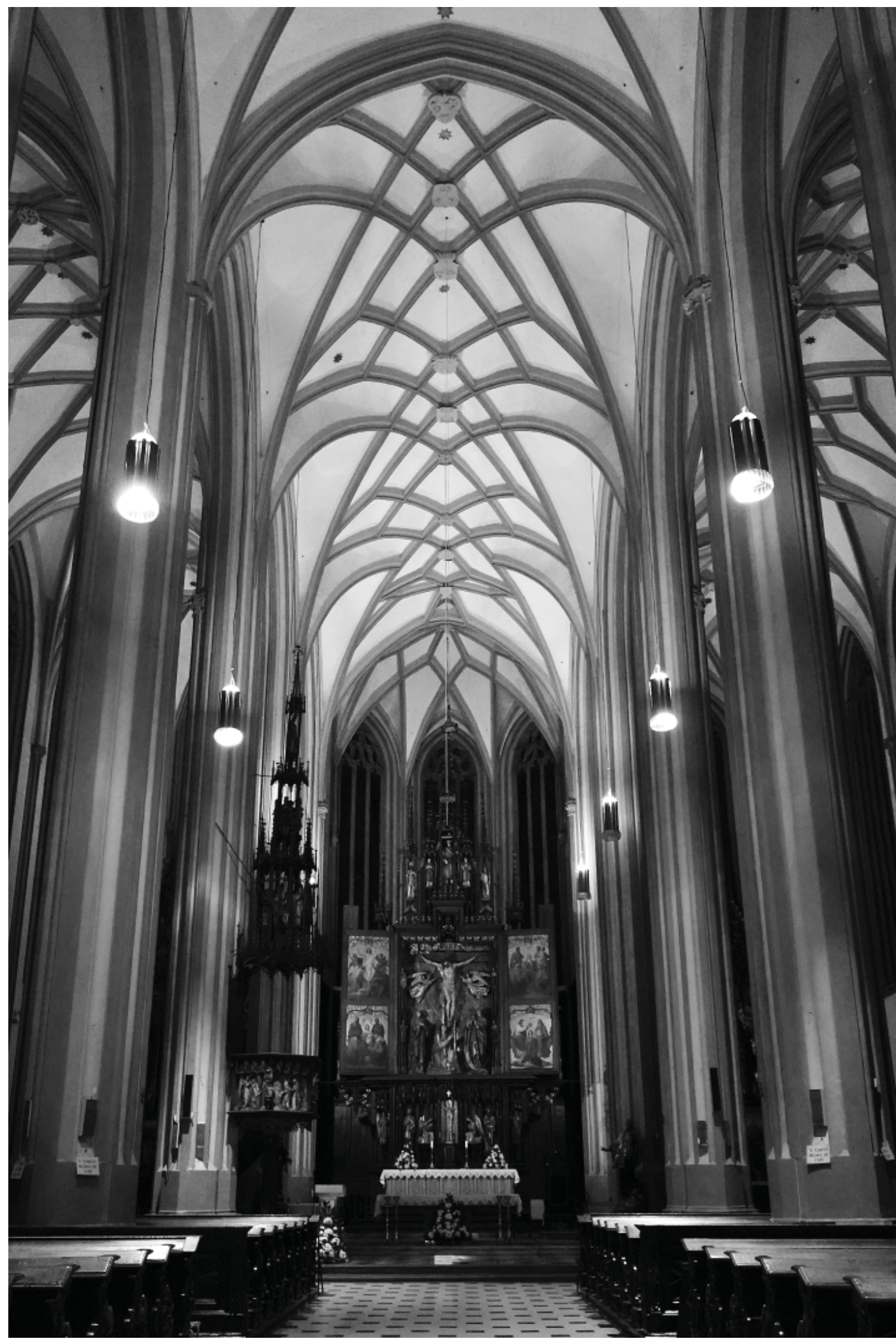

Abb. 1: Olmütz (Olomouc), St. Moritz, Chorinnenraum. Foto: Kristina Kogler 
Abb. 2: Olmütr, (Olomouc), St. Moritz, maßstabsgerechter Grundriss nach Naturmaß. Zeichnung: Jakob Kremsner, auf Basis des Vermessungsplans von Ing. Alois Steflea

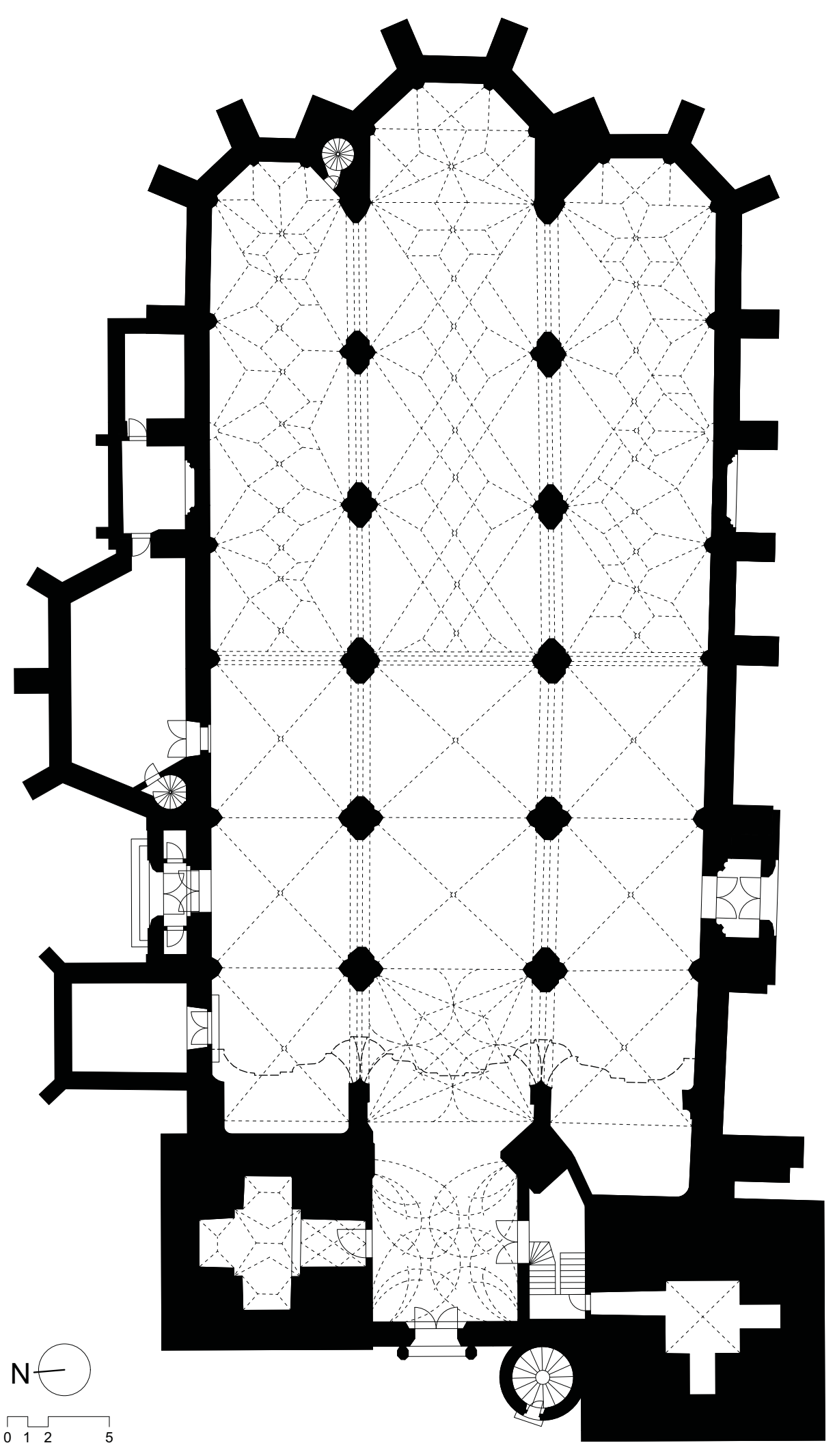



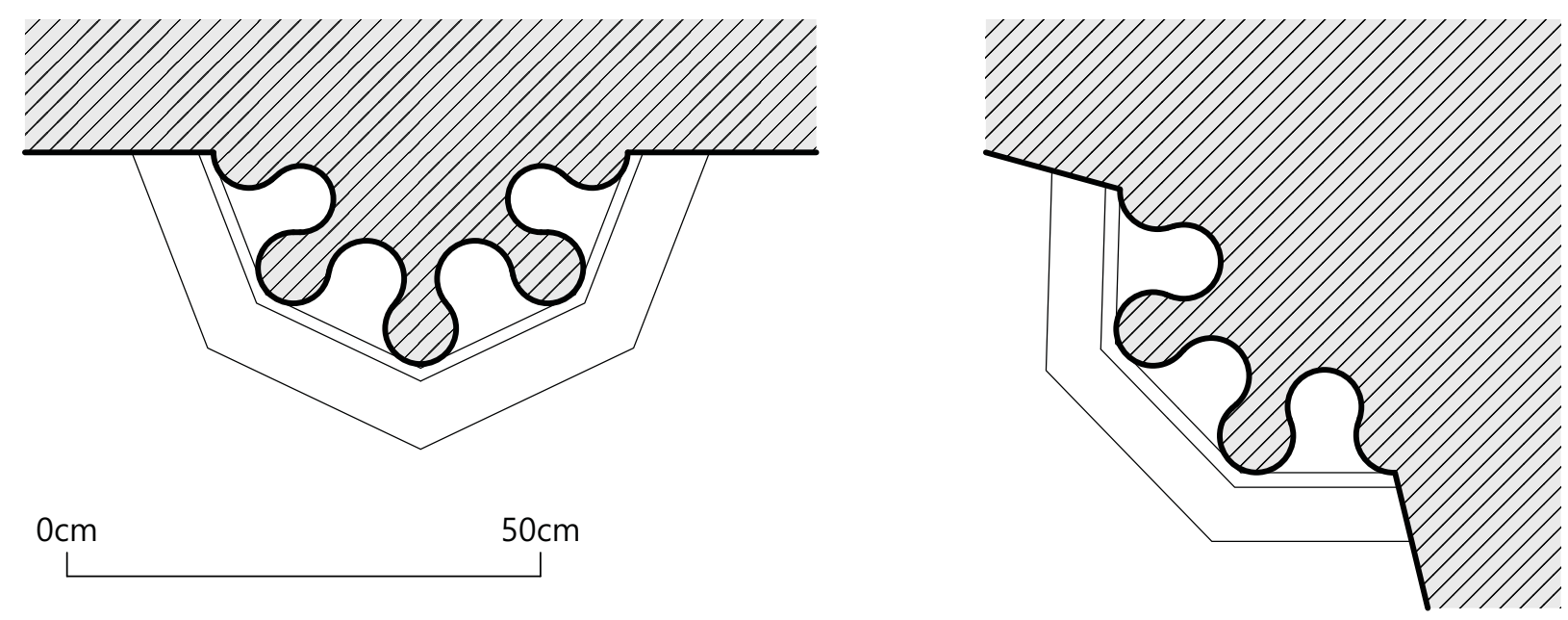

Abb. 3: Olmütz, (Olomouc), St. Moritz, schematische Querschnitte zweier Wandvorlagen im südlichen Seitenschiff. Zeichnung: Jakob Kremsner

fungen erklärt. ${ }^{2}$ Und das zu Unrecht: Denn die von eleganten Wand- und Pfeilervorlagen aufsteigenden Netzfigurationen des östlichen Hallenchors stehen ihren nachfolgenden Artgenossen immerhin ebenso wenig nach, wie die Formensprache der hier realisierten Einbauten denjenigen im Langhaus. Ganz im Gegenteil betonen die differenziert realisierten Laubblattkapitelle, verästelten Portalrahmungen, spannungsreichen Maßwerkentwürfe und bereits

befindet sich im Archiv des NPÚ ÚOP v Olomouci (spisovna dokumentačních fondů, inv. č. 1188) und wurde freundlicherweise von Ondřej Belšík zur Verfügung gestellt. Zu den beiden Planvarianten, die bisher in der Forschungsliteratur verwendeten wurden, siehe die Publikationen von Adolph Nowak und August Prokop (wo die Pläne das erste Mal verwendet wurden): NOWAK, A.: Kirchliche Kunst-Denkmale aus Olmütz, Bd. 1. Olomouc 1890, S. 5 (Abb. 6); PROKOP, A.: Die Markgrafschaft Mähren in kunstgeschichtlicher Beziehung: Grundzüge einer Kunstgeschichte dieses Landes mit besonderer Berücksichtigung der Baukunst, Bd. 2. Brno 1904, S. 428 (Abb. 586).

2 Dies zeigt sich sowohl in der Schwerpunktsetzung der zum Kirchenbau erschienenen Artikel, als auch in der Ausführlichkeit, in welcher die Weststeile im Gegensatz zum Chor innerhalb der jüngeren Überblickwerke behandelt wurden. Siehe u.a. MYSLIVEČOVÁ, H.: Pracovala hut'Benedikta Rieda v Olomouci? In: Historické Olomouc, Bd. 6. Olomouc 1992, S. 115121; BLÁHOVÁ, Z.: Interpretace architektonických vzorů pražské a vídeňské huti v olomouckých stavbách přelomu 15. a 16. století (v kapli sv. Jeronýma a v kostele sv. Moŕice). In: Doba Jagellonskáv żemích české koruny (1471-1526). Konference k erwähnten Gewölbefigurationen, dass es sich beim Chorprojekt um eine äußerst anspruchsvolle Architektur handelt - und zwar von internationalem Rang.

In der langen Forschungsgeschichte des Kirchenbaus blieb dies nicht unbemerkt. So waren neben Jan Sedlák auch Ivo Hlobil und Zdenka Bláhová um die Betonung baukünstlerischer Verbindungen zu maßgebenden Bauprojekten der Zeit (wie etwa die Pfarrkirchen in Brünn [Brno $]^{3}$ und Wien ${ }^{4}$ ) bemüht,

založeni Ústavu dějin kerest'anského Umèni KTF UK v Praže (2.-4. 10. 2003). Ed.: KUBÍK, V. České Budějovice 2005, S. $347-$ 362; HLOBIL I. - TOGNER M. - HYHLÍK V.: Olomouc. Probstei- und Pfarrkirche St. Mauritz. (= Kirchliche Denkmäler, 10). Velehrad 1992, S. 3-11; BLÁHOVÁ Z.: Kostel sv. Morice. 1412-1540. Olomouc. In: Od gotiky ke renesanci. Výtvarná kultura Moravy a Slezska 1400-1550, Olomouc, Bd. 3. Eds.: HLOBIL, I. - PERU゚TKA, M. [Kat. Ausst.]. Olomouc 1999, S. 204-208; BENEŠOVSKÀ, K. et al.: Architecture of the Gothic (= Ten Centuries of Architecture, 2). Prague 2001, S. 265.

3 St. Jakob, 1446-1530. Siehe KROUPA, J. - ŠEFERISOVÁ, L. - JAKUBEC, O.: Chrám sv. Jakuba. In: Dẹjiny Brna 7. Umèleckohistorické památky. Historické jádro. Ed.: KROUPA, J. Brno 2015, S. 261-270; KROUPA, P.: Farní Kostel sv. Jakuba Většího v Brne. Brno. In: Od gotiky k renesanci. Výtvarná kultura Morary a Slezska 1400-1550. Brno, Bd. 2. Eds.: HLOBIL, I. PERUTKA, M. [Kat. Ausst.]. Brno 1999, S. 90-96; PROKOP 1904 (wie Anm. 1), S. 432-438, 474-476, 502-503, 566-571; SAMEK, B.: Umĕlecképamátky Moravy a Slezkea, Bd. 1. Prag 1994, S. 164; BRETHOLZ, B.: Die Pfarrkirche St. Jakob in Brünn. Brno 1901, S. 78-81; BENEŠOVSKÁ 2001 (wie Anm. 2), S. 265. 
wobei letztere ebenso für eine - fast schon konventionelle - Ableitung von parlerischen Formschöpfungen plädierten. ${ }^{5}$ Unabhängig davon verwiesen auch Norbert Nussbaum und Sabine Lepsky auf formale Analogien zwischen der Olmützer und der Brünner Pfarrkirche sowie den Entwürfen der Wiener Bauhütte. ${ }^{6}$ Betont sei jedoch, dass es sich bei diesen Beobachtungen lediglich um knappe Randbemerkungen handelt, die keinen Nachhall fanden und der fest verankerten Bewertung des Kirchenbaues nicht entsprechend entgegenwirken konnten. Angesichts dieses Defizites soll im Folgenden die Frage aufgearbeitet werden, wie der Olmützer Hallenchor von seiner künstlerischen sowie typologischen Gestalt in das Geflecht der regionalen, aber vor allem auch mitteleuropäischen Architekturentwicklung einzuordnen ist.

Ausgangspunkt für meine Auseinandersetzung mit dem kunsthistorischen Stellenwert des Olmützer Hallenchors bildet dessen zeitliche Einordnung. In der Forschungsliteratur hat sich die Haltung durchgesetzt, den Chor als Ergebnis einer Baukampagne anzusehen, welche erst nach der Vollendung des Langhauses zwischen den Jahren 1453 und 1483 bzw. 1492 verwirklicht wurde. ${ }^{7}$ Eine derartige Datierung des gesamten Baukörpers ist aber keineswegs nachvollziehbar. Denn der Grundriss vom St. Moritz (Abb. 2) zeigt deutlich an, dass die Umfassungsmauern und Freipfeiler nicht demselben Plankonzept entspringen: Immerhin steht die äußere durch die Strebepfeiler gegebene Jochteilung mit der inneren, die durch die Mittelschiffpfeiler angegeben wird, im Widerspruch. Selbst auf stilistischer Ebene wird eine entsprechende Differenzierung greifbar:

\footnotetext{
4 St. Stephan, Langhaus, ca. 1400-1460. Siehe SCHEDL, B.: St. Stephan in Wien. Der Bau der gotischen Kirche (1200-1500). Wien - Köln - Weimar 2018, S. 93-124; BÖKER, J. J.: Der Wiener Stephansdom. Ein Sinnbild des Hauses Österreich. Salzburg 2007, S. 157-175; JUCKES, T.: Gewölbe der Stephanskirche. Wien als Architekturzentrum in Mitteleuropa. In: Wiener Jabrbuch für Kunstgeschichte, 62, 2014, S. 42-43.
}

5 SEDLÁK, J.: Zum Problem der Datierung und des Stilcharakters des Presbyteriums der St.-Jakob-Pfarrkirche in Brünn. In: Sborník praci filosofické Fakulty Brnenské University, 19-20, Brno 1971, S. 148-153; BLÁHOVÁ 1999 (wie Anm. 2), S. 204-205. Auf die Verwandtschaft zwischen dem Ölmützer und Brünner Chorgewölbeentwurf hat bereits August Prokop hingewiesen, aber nicht tiefergreifend thematisiert. Siehe

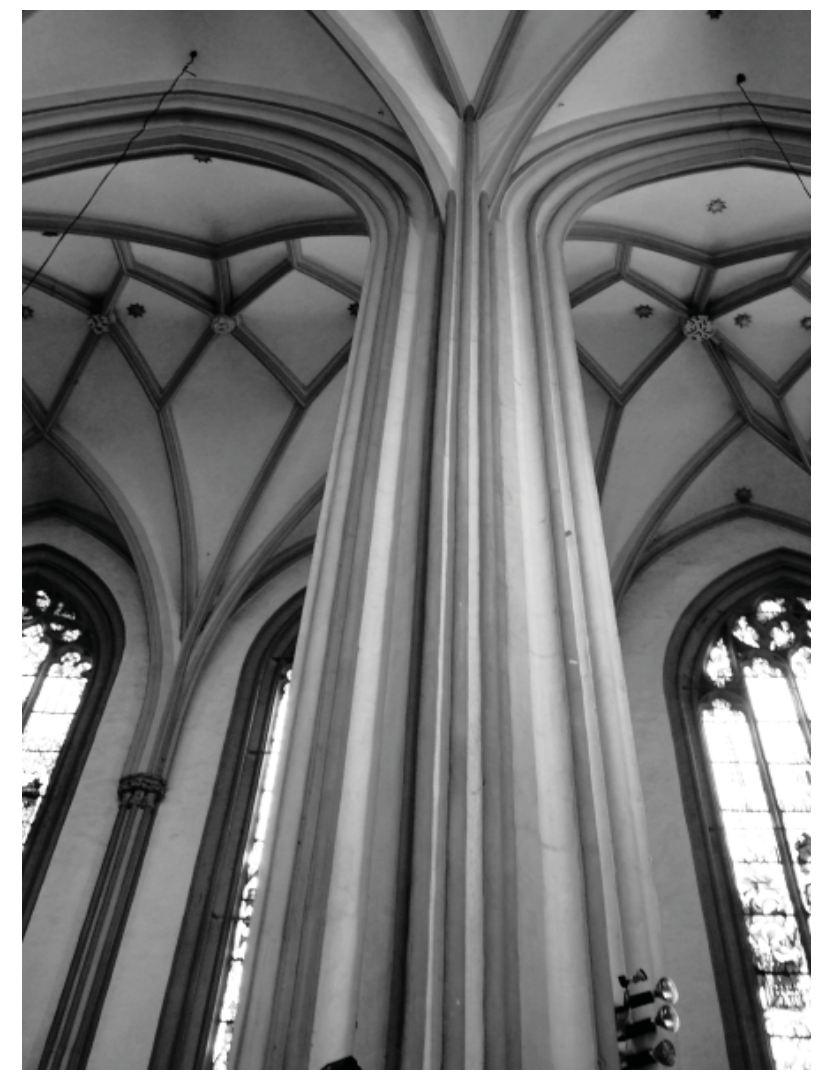

Abb. 4: Olmütz,(Olomouc), St. Mority, Chor, Blick vom Mittelschiff in das nördliche Seitenschiff. Foto: Sophie Morawitz.

denn die Dienstsysteme der Außenwände (Abb. 3-4) und Freipfeiler (Abb. 4-5) folgen zwei grundverschiedenen ästhetischen sowie architektonischen Auffassungen.

PROKOP 1904 (wie Anm. 1), S. 558.

6 NUSSBAUM, N. - LEPSKY S.: Das gotische Gewölbe. Eine Geschichte seiner Form und Konstruktion. Darmstadt 1999, S. 370 (Anm. 896). Siehe auch: NUSSBAUM, N.: Deutsche Kirchenbaukunst der Gotik. Darmstadt 1994, S. 235.

NOWAK 1890 (wie Anm. 1), S. 1-12; MATZKE, J.: Die St. Mauritzkirche in Olmüt\%. Das Bawwerk, die Einrichtung und die Pfarrerreibe. Steinheim am Main 1964, S. 7; HLOBIL - TOGNER - HYHLÍK 1992 (wie Anm. 2), S. 7; BENEŠOVSKÁ 2001 (wie Anm. 2), S. 252; BLÁHOVÁ 2005 (wie Anm. 2), S. 347-362; RYDLOVÁ, M.: Rakousko-podunajské paralely pozdně gotické sakrálni architektury na Moravé, Masterarbeit (unpubl.). Olomouc 2014, S. 15-30. 


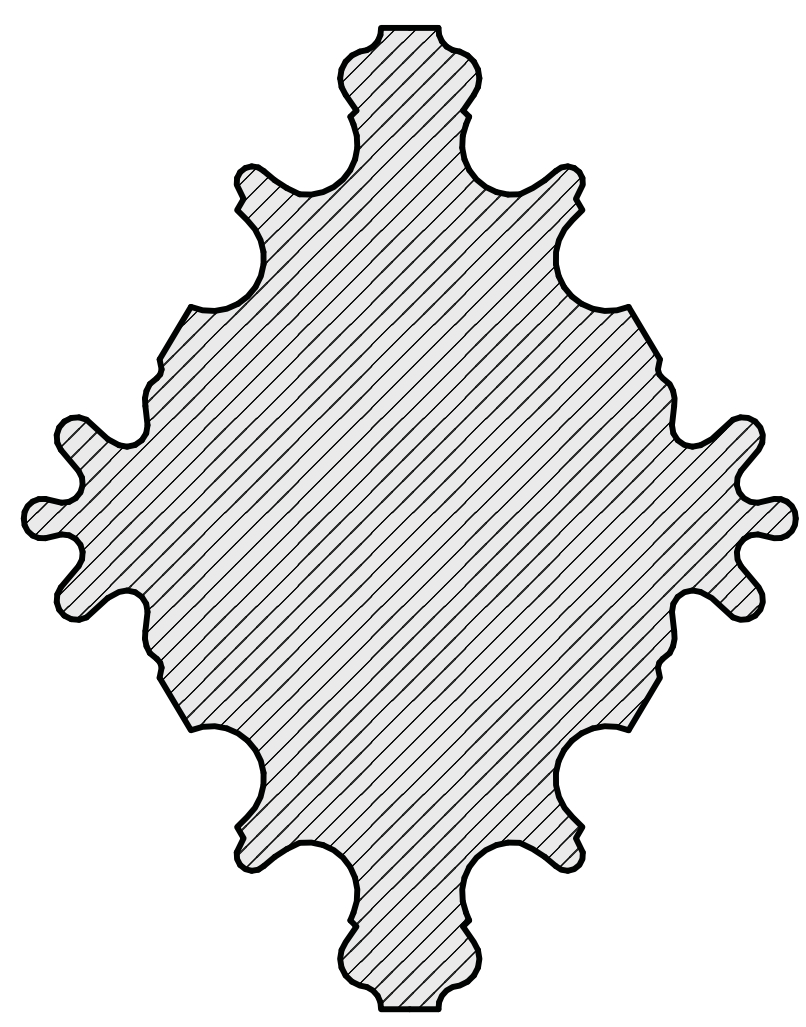

Abb. 5: Olmütz, (Olomouc), St. Moritz, Chor, schematischer Querschnitt eines Freipfeilers. Zeichnung: Jakob Kremsner (auf Basis des bei MATZKE, J.: Die St. Moritz-Kirche in Olmütr. Das Bawwerk, die Einrichtung und die Pfarrerreihe. Steinheim am Main 1964 publizierten Querschnittes)

So zeigen die Umfassungsmauern des im Grunde gestaffelten, dreiapsidialen Hallenchors eine Gliederung in vier enggestellte Joche, die im Inneren runde, durch weiche Kehlen zusammengefügte Dienste gleicher Dimension besitzen (Abb. 3-4). Diese enden in Laubkapitellen, auf welchen die heruntergeleiteten Rippenläufe des Gewölbes ruhen. Ganz anders präsentiert sich indessen das System der Freipfeiler (Abb. 4-5). Zum einen bilden die rhombischen Arka-

8 PROKOP 1904 (wie Anm. 1), S. 431-432.

9 Ibidem, S. 429, 431-432. Zum Kirchenbrand von 1453 siehe WOLNY, G.: Kirchliche Topographie von Mähren. Meist nach Urkunden und Handschriften. Brno 1855, S. 225; WOLNY, G.: Die Markgrafschaft Mähren. Topographisch, statistisch und historisch geschildert. Olmützer Kreis, Bd. 5. Brno 1846, S. 75; NOWAK 1890 (wie Anm. 1), S. 2; HLOBIL - TOGNER - HYHLÍK denpfeiler drei Joche aus, welche in keinerlei Relation zu den vier Jochen der Chorumfassungsmauern stehen, und zum anderen unterscheidet sich das hier angewandte Dienstsystem in mehreren Punkten von jenem der Hochschiffwände. Während die mittelschiffseitigen Dienste kämpferlos in Richtung Gewölbe hinauflaufen und die herunterkommenden Rippenläufe in senkrechter Linie durchstoßen, weisen die Rundstäbe der Abseiten zwischengeschaltete Kapitellblöcke auf. Doch von einem Tragen der Dienste und einem Lasten der Gewölberippen kann auch hier keine Rede sein, da die Rundstabprofile der Dienste über den Bündelkapitellen in ihrem vertikal nach oben strebenden Verlauf weitergeführt werden und die Rippenanfänger aus den Zwischenräumen der Dienste bzw. den Gewölbefüllungen herauswachsen. Hinzu kommt, dass die Bündelkapitelle der Arkaturen erheblich höher sitzen als jene an den Umfassungsmauern und hinsichtlich Differenzierung und Ausdehnung eine Steigerung erfahren.

Demnach werden die Umfassungsmauern und Pfeiler sowohl durch eine Abweichung des Modulsystems, als auch durch den Gegensatz zwischen einem der althergebrachten tektonischen Ordnung folgenden Dienstsystem auf der einen, und nahezu linearen Vorlagen auf der anderen Seite bestimmt. Und diese Erkenntnis ist keineswegs neu: Bereits im beginnenden 20. Jahrhundert erkannte August Prokop die Abweichung der äußeren und inneren Pfeilerstellung und interpretierte diese im Sinne eines zeitlichen Unterschiedes in der Entstehung. ${ }^{8}$ Dabei setzte er den Anfang der Arbeiten in das letzte Drittel des 14. Jahrhunderts und den Innenausbau der Chorhalle in die Zeit nach dem Kirchenbrand von 1453. ${ }^{9}$ Von Bohumil Zlámal noch bestätigend übernommen, wurde Prokops These einer mehrphasigen Ausführung von den nachfolgenden Generationen allerdings zugunsten einer homogenen Bauchronologie in Nachfolge von Adolf Nowak, ${ }^{10}$ verworfen - ja gar ignoriert. Doch angesichts der

1992 (wie Anm. 2), S. 7; PROKOP 1904 (wie Anm. 1), S. 429, 431. Bei Alois V. Šembera wird abweichend 1451 als Jahr des Stadtbrandes genannt. Siehe ŠEMBERA, A. V.: Paméti a znamenitosti mèsta Olomouc. Vídeň 1861, S. 59-60.

${ }^{10}$ Zur Baugeschichte nach Adolf Nowak siehe NOWAK 1890 (wie Anm. 1), S. 1-12. 


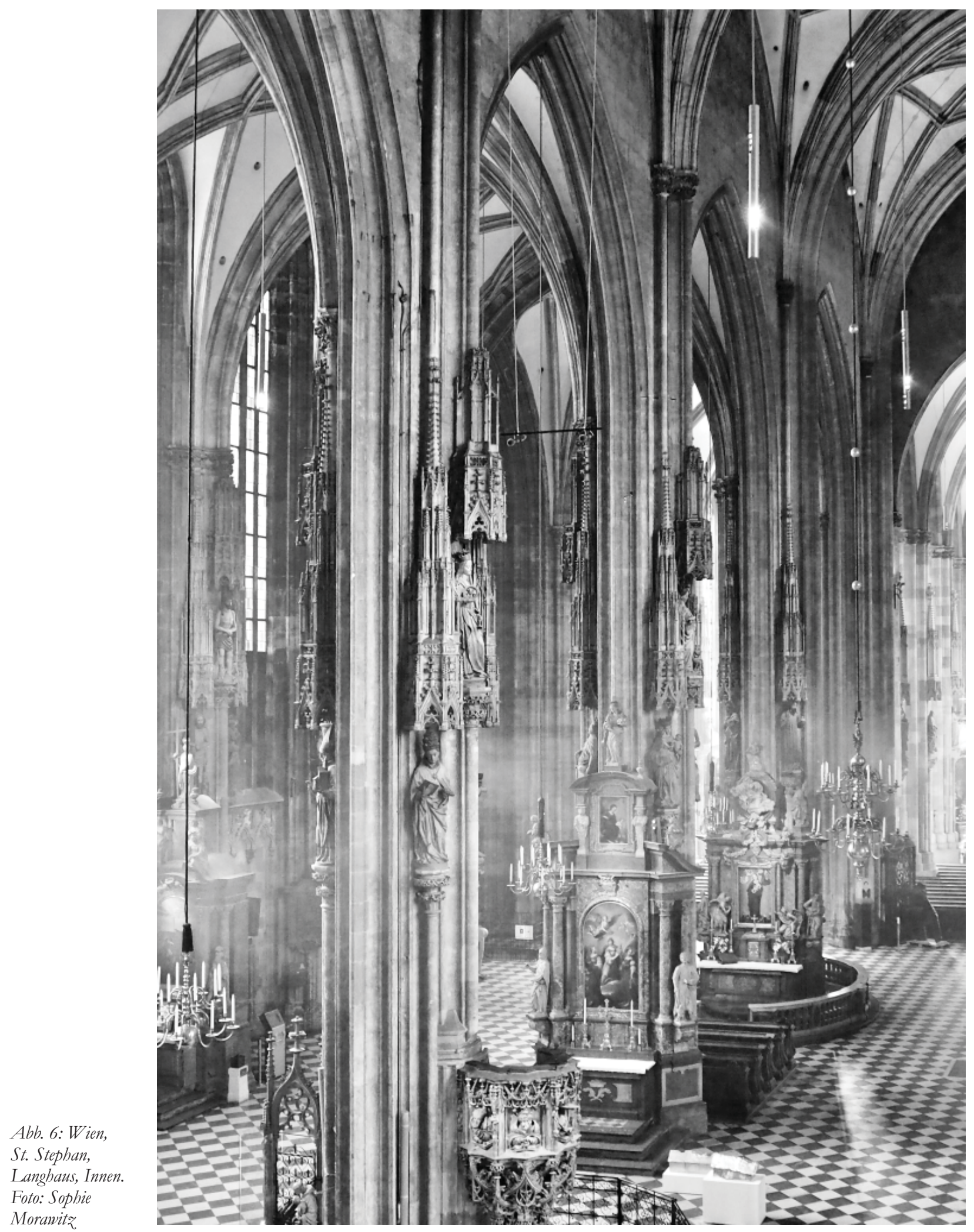


eben beschriebenen stilistischen sowie formalen (!) Differenz muss die Frage gestellt werden, inwieweit Prokops Interpretationen und Datierungsansätze aus der Vergessenheit geholt werden müssen. Um das beantworten zu können, soll im Folgenden die Herkunft der beiden kontrastierenden Gestaltungsweisen und deren Datierung hinterfragt werden.

Was die Olmützer Bündelpfeiler betrifft, fügt sich ihr Design auch tatsächlich in die bisher vorgeschlagene ostmitteleuropäische Architekturentwicklung der 2. Hälfte des 15. Jahrhunderts. ${ }^{11}$ Auch wenn eine entsprechende Kombination aus ondulierend verschleifenden Profilabfolgen und in Längsrichtung vorgelagerten sog. gedrückten Birnstäben ${ }^{12}$ bereits ab der Mitte des 14. Jahrhunderts - und zwar vor allem in der böhmischen Architektur - greifbar wird, ${ }^{13}$ lässt sich eine weitaus auffälligere Parallele zu diversen, in der 2. Hälfte des 15. Jahrhunderts errichteten, Pfeilerentwürfen aus dem Wirkungsfeld der Wiener Bauhütte schlagen: Nämlich den Langhauspfeilern der Wiener Stephanskirche (ca. 1430-1440; Abb. 6), den Arkaden des Chors der Steyrer Stadtpfarrkirche (wohl 3. Viertel 15. Jhdt.) sowie den Stützen im Langhaus der Eggenburger Pfarrkirche (um 1489). ${ }^{14}$ Bei all diesen handelt es sich um plastisch durchwirkte Bündelpfeiler, deren Kern hinter ondulierenden Vorlagengruppen vollkommen verschwindet. Dabei laufen die in Längsrichtung vorgesetzten Birnstäbe durch äußerst weitgespannte Scheidbögen zum korrespondierenden Pfeiler hinüber, während die

\footnotetext{
${ }^{11}$ Siehe oben, Anm. 7 sowie PROKOP 1904 (wie Anm. 1), S. 429, 432, 571-573.
}

12 Zum Terminus des gedrückten Birnstab bzw. stlačený hruškovec siehe: ADAMSKI, J.: Böhmische Einflüsse in der schlesischen Kirchenbaukunst des mittleren 14. Jahrhunderts. Der Fall Schweidnitz und Striegau. In: Umèni Art, 65, 2017, Nr. 4, S. 339, 345 (Anm. 12).

${ }^{13}$ Zu den böhmischen Beispielen mit gedrückten Birnstäben, wie z.B. den Pfeilern in der Augustinerkirche $\mathrm{Na}$ Karlově in Prag (Praha) und der Zisterzienserkirche in Skalitz (Skalice) siehe: ADAMSKI 2017 (wie Anm. 12), S. 338-339.

${ }^{14}$ Auf die Ähnlichkeiten zwischen den Olmützer Stützen und den zeitgleichen Lösungen des österreichischen, aber auch böhmischen, Raumes hat bereits Wulf Schadendorf hingewiesen, obgleich er auf die Benennung konkreter Vergleichsbeispiele verzichtete. Norbert Nussbaum leitete

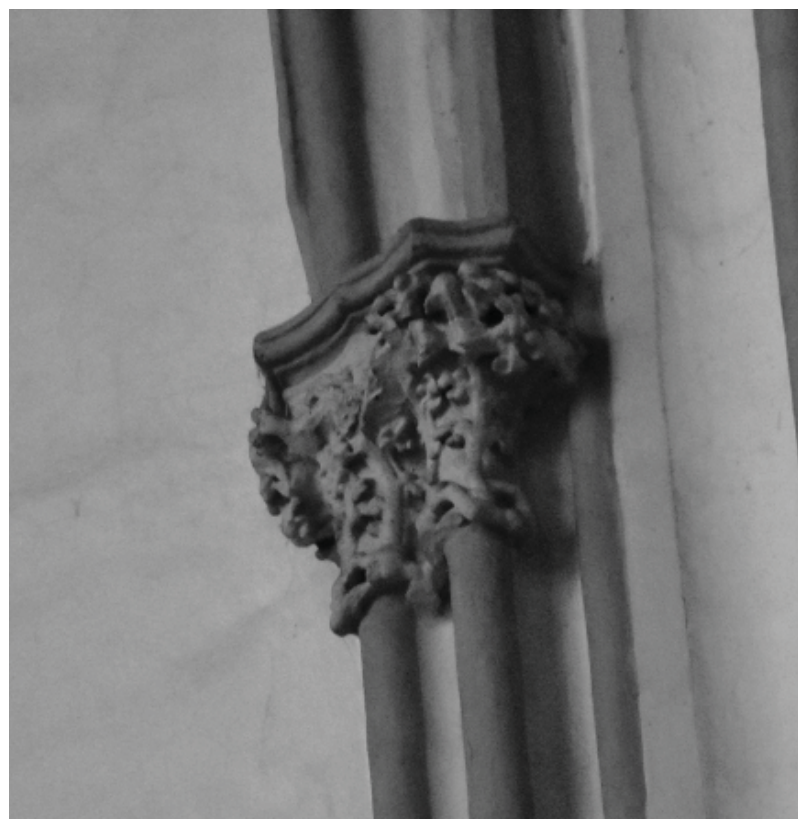

Abb. 7: Olmütr, St. Morit», Kapitell im nördlichen Chorpolygon. Foto: Die Bauforscher (Oliver Fries/Lisa-Maria Gerstenbauer)

schiffseitigen Dienstbündel direkt in die Gewölbezone hinaufgeleitet werden. Selbst die für Olmütz so typischen Dienstbündeln, die den Scheidarkaden lediglich vorgesetzt sind und eine laibungsartige Erscheinung dieser erzeugen, sind in Wien, Steyr und Eggenburg ebenfalls verwirklicht. ${ }^{15}$ Dass sich

die Olmützer Stützen direkt von den Lösungen in Wien und Steyr ab; das Eggenburger Langhaus wurde in Zusammenhang dessen bisher aber nicht als Vergleich herangezogen. Dazu siehe SCHADENDORF, W.: Wien, Prag und Halle. Ein Beitrag zum Einfluß der Dombauhütten von Wien und Prag auf die Baukunst Mitteldeutschlands, dargestellt an Chor und Langhaus von St. Moritz in Halle/Saale. In: Hamburger Mittel- und Ostdeutsche Forschungen. Kulturelle und wirtschaftliche Studien in Beziebung zum gesamtdeutschen Raum, 3, 1961, S. 187; NUSSBAUM 1994 (wie Anm. 6), S. 235. Zur Datierung der Stützsysteme in Wien, Steyr und Eggenburg siehe: BÖKER 2007 (wie Anm. 4), S. 162-166; BÖKER, J. J.: Der Chor der Stadtpfarrkirche von Steyr und seine Baumeister. In: Österreichische Zeitschrift für Kunst und Denkmalpflege, 57, 2003, S. 215; Debio-Handbuch: Die Kunstdenkmäler Österreichs. Niederösterreich nördlich der Donau. Wien - Horn 1990, S. 150.

${ }^{15}$ Dabei ist zu unterstreichen, dass die Dienstbündel in Wien und Steyr in Form von Birnstäben ausgeführt wurden. Dass 


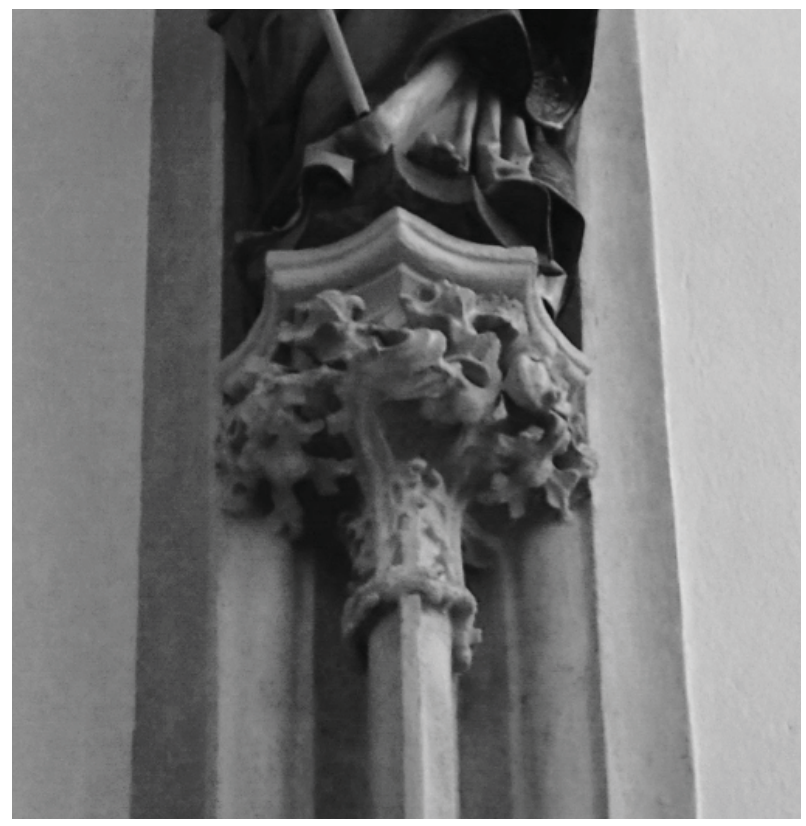

Abb. 8: Brünn (Brno), St. Jakob, Kapitell an der nördlichen Hochschiffwand des Chores. Foto: Sophie Morawitz.

in Wien zudem an mehreren Standorten Kapitelle finden, die mit ihren vegetabilen Dekor und polygonalen Deckplatten mit denjenigen in den Olmützer Chorseitenschiffen und -polygonen eng verwandt sind (Abb. 7; neben dem Langhaus auch am sog.

die Olmützer Arkaturen in Abweichung dazu Rundstabdienste aufweisen, erklärt sich in Abstimmung mit dem Dienstsystem der Umfassungsmauern, welche ebenfalls sehr schlanke Rundstabdienste abweisen.

${ }^{16}$ Die Errichtung dieser Bereiche und Einbauten ist, neueren Ergebnissen zufolge, in die 1460er bis 1480er Jahre zu setzten. Interessant in diesem Zusammenhang ist zudem eine Skulpturenkonsole, welche sich am dritten Arkadenpfeiler des Langhauses befindet und weitreichend mit dem Olmützer Beispielen in Übereinstimmung zu bringen ist. Da Johann J. Böker die Skulpturenausstattung der Freipfeiler in das 3. Viertel des 15. Jahrhunderts setzt, kann für diese eine Errichtung in der Folgezeit vorausgesetzt werden. Zur Datierung der genannten Beispiele siehe: BÖKER 2007 (wie Anm. 4), S. 227-235, 239-245.

17 Dehio-Handbuch: Die Kunstdenkmäler Österreichs. Wien I. Bezirk - Innere Stadt. Horn - Wien 2007, S. 96, 98; HASSMANN, E.: Meister Michael. Baumeister der Heræöge von Österreich. Wien - Köln - Weimar 2002, S. 352.
Puchheimbaldachin, der Barbarakapelle und der Nordturmvorhalle oder dem Stiegenhäuschen der sog. Singergalerie) $)^{16}$, hebt die dargelegte Beziehung abermals hervor.

Die Verwandtschaft zwischen den Olmützer Stützen und dem Formenrepertoire der Wiener Hütte wird dann noch augenfälliger, wenn man zeitgleiche Kapitellausformungen von Bauprojekten mit in den Blick fasst, die mit den Schöpfungen in Wien in Verbindung zu bringen sind. So zeichnen sich sowohl diverse Kapitelllösungen der Wiener Kirche Maria am Gestade (Langhaus, 1394-1414, und v.a. Empore, ab 1414 $)^{17}$ durch ein Geflecht aus vertikal kriechenden Buckellaub und abschließenden Deckplatten mit konkaven Ausschwüngen aus, als auch jene am Sakramentshäuschen im Chor der Steyrer Stadtpfarrkirche $(1470 \mathrm{er})^{18}$, der Westempore der Nikolauskirche in Znaim (Znojmo; nach 1442) ${ }^{19}$ und des Chors von St. Jakob in Brünn (Brno; 1446 bis etwa 1473; Abb. 8) ${ }^{20}$. Dabei müssen besonders der letztgenannten Lösung aufgrund ihrer formalen Deckung, aber auch den ident gestalteten virtuosen Gewölbeanfängern, Implikationen für die Datierung der Olmützer Kapitelle zugeschrieben werden.

Diese zeitliche Ansetzung der Freipfeiler deckt sich zudem mit den wenigen erhaltenen Quellentexten: Während die Stiftung von 200 Gulden durch Martin Knewsl „[...] zum Ausbau der Pfeiler der Mauritzkirche [...]. ${ }^{“ 21}$ aus dem Jahre 1435 einen

${ }^{18}$ BÖKER 2003 (wie Anm. 13), S. 226-227. Mit den Kapitellen in Olmütz sind in den österreichischen Erblanden zudem die fragmentarisch erhaltenen Bündelkapitelle der Pfarrkirche in Mödling (1454-1523/25) eng verwandt. Zu dieser siehe: Dehio-Handbuch: Die Kunstdenkmäler Österreichs. Niederösterreich südlich der Donau, Bd. 2. Wien - Horn 2003, S. 1459-1463.

${ }^{19}$ BÖKER 2007 (wie Anm. 4), S. 214; BENEŠOVSKÁ 2001 (wie Anm. 2), S. 267; BUREŠ, J.: Die Meister des Pressburger Domes. In: Acta Historiae Artium. Academiae Scientiarum Hungaricae, 18, 1972, S. 98.

${ }^{20}$ BUREŠ 1972 (wie Anm. 18), S. 100; BENEŠOVSKÁ 2001 (wie Anm. 2), S. 265; SEDLÁK 1971 (wie Anm. 5), S. 149.

${ }^{21}$ NOWAK 1890 (wie Anm. 1), S. 2. Diese Quelle findet sich zudem bei: PROKOP 1904 (wie Anm. 1), S. 429, 432; HLOBIL - TOGNER - HYHLÍK 1992 (wie Anm. 2), S. 5; KUX, J.: Geschichte der königlichen Hauptstadt Olmütz bis zum Umstury. 1918 (= Sudetendeutsche Stadtgeschichten, 1). Liberec Olomouc 1937, S. 109. 
terminus a quo für die Errichtung der Arkadenreihen vorgibt, ${ }^{22}$ liefert die am westlichsten Schlussstein des Mittelschiffgewölbes angebrachte Jahreszahl 1483 einen terminus ad quem. ${ }^{23}$

Weitaus verblüffender als die Frage nach der Entstehungszeit der Freipfeiler gestaltet sich die Suche nach dem System, welches die Umfassungsmauern prägt. Erweitert man die vier Jocheinheiten der Hochschiffwände auf entsprechende Arkadenpfeiler, ergibt sich ein modulares Prinzip, welches auf schmalen, querrechteckigen Dimensionen basiert. Und dieses ist nicht nur erheblich konventioneller als die nahezu quadratischen Joche der Stützen, sondern entspricht einem Anordnungsschema, welches die Sakralbauten des 13. und 14. Jahrhunderts - seien es die klassischen französischen Kathedralen oder deren mitteleuropäische Nachläufer - bestimmt.

In Übereinstimmung damit stand auch die dreiapsidiale Hallenchorlösung bereits seit dem 14 . Jahrhundert zur Verfügung. ${ }^{24}$ Dabei deutet vieles darauf hin, dass das in Olmütz angewandte Zusam-

\footnotetext{
${ }^{22}$ Die Überlieferung zum Bau der Pfeiler wurde (mit Ausnahme von August Prokop) auf die Bauarbeiten am Langhaus bezogen. Bricht man die Datierung des Chores in die 2. Hälfte des 15. Jahrhunderts auf, ergibt sich allerdings die Möglichkeit, die erwähnte Stiftung ebenfalls auf den Ausbau des Chors (bzw. auf Chor und Langhaus gleichermaßen) zu beziehen. Siehe BENEŠOVSKÁ 2001 (wie Anm. 2), S. 267; BLÁHOVÁ 1999 (wie Anm. 2), S. 204-207; HLOBIL - TOGNER - HYHLÍK 1992 (wie Anm. 2), S. 5; NOWAK 1890 (wie Anm. 1), S. 2-3; Zu Prokop siehe: PROKOP 1904 (wie Anm. 1), S. 429, 432; 571-573.
}

${ }^{23}$ Das erkannten auch: BENEŠOVSKÁ 2001 (wie Anm. 2), S. 252; HLOBIL - TOGNER - HYHLÍK 1992 (wie Anm. 2), S. 7; NOWAK 1890 (wie Anm. 1), S. 3; PROKOP 1904 (wie Anm. 1), S. 429, 571-573.

${ }^{24}$ Als frühe Beispiele dreiapsidialer Hallenchöre im mitteleuropäischen Raum sind die Lösungen der Pfarrkirche St. Maria zur Wiese in Soest (ab 1313), der Marktkirche St. Georgii et Jacobi in Hannover (ab 1335 [ältere Forschung] bzw. nach 1290 [Johann J. Böker]), der Wiener Stephanskirche (ab den 1320er) und der steirischen Wallfahrtskirche Maria Straßengel bei Graz (1346-1355) zu nennen. All diese Staffelchoranlagen besitzen jedoch Lösungen, welche von der in Olmütz realisierten Variante in mehreren konstruktiven sowie gestalterischen Aspekten abweichen. Siehe NUSSBAUM 1994 (wie Anm. 6), S. 129-130; 361 (Anm. 292); SCHADENDORF, W.: Die Marktkirche zu Hannover (= Kleine Kunstführer für Niedersachsen, 7). Göttingen 1954, S. 2; BÖKER, J. J.: Die menspiel aus einem 5/8-Schluss im Mittelschiff und 3/8-Polygonen in den Seitenschiffen im 2. Viertel des 14. Jahrhunderts im Herzogtum Schlesien entwickelt wurde. Als wohl ältestes erhaltenes Beispiel entsprechender Disposition ist der Staffelchor der Pfarrkirche St. Maria auf dem Sande in Breslau (Wrocław; Abb. 9/a) zu nennen. Dieser wurde im Jahre 1334 grundgelegt und konnte den Schriftquellen zufolge bereits 1369 bespielt werden. ${ }^{25}$ Nur kurze Zeit später erfolgte in Breslau ab den 1340er Jahren zudem die Errichtung des Ostschlusses der Elisabethkirche nach gleichem (wenn auch basilikalem) Entwurf (Abb. 9/b). ${ }^{26}$ Beide Bauten bestechen durch eine zentrale 5/8-Apside, welche unmittelbar in der Höhe der flankierenden 3/8-Polygone anschließt; obgleich die Schwierigkeiten, die bei der Sandkirche noch in Bezug auf das Verhältnis der drei Chorschlüsse zueinander zu beobachten sind, im Falle der Elisabethkirche bereits durch die Ausbildung massiver Mauerkeile im Zwickel zwischen Hauptund Nebenchor korrigiert werden konnten. ${ }^{27}$ Dabei

Markthalle in Hannover. Zur zeitlichen Stellung der gotischen Backsteinhalle. In: Niederdeutsche Beiträge zur Kunstgeschichte, 25, 1986, S. 33-46; LUKAS, V.: St. Maria zur Wiese. Ein Meisterwerk. gotischer Baukunst in Soest. München/Berlin 2004, S. 16; BOKER 2007 (wie Anm. 4), S. 45-46; SCHEDL 2018 (Wie Anm. 4), S. 39-48; KOCH, R.: Die Baugeschichte der Stadtpfarrkirche von Steyr im Mittelalter. In: KOCH, R. - PROKISCH, B.: Stadtpfarrkirche Steyr. Baugeschichte und Kunstgeschichte. Steyr 1993, S. 40; WAGNER-RIEGER, R.: Gotische Architektur in der Steiermark. In: Gotik in der Steiermark [Kat. Ausst.]. Graz 1978, S. 65-66.

${ }^{25}$ Mittelalterliche Architektur in Polen. Romanische und gotische Baukunst zwischen Oder und Weichsel, Bd. 2. Hrsg. von Ch. HERMANN - D. VON WINTERFELD. Petersburg 2015, S. 618-620; BURGEMEISTER, L.: Die Kunstdenkmäler der Stadt Breslau, Bd. 1 (= Die Kunstdenkmäler der Provinz Niederschlesien, 1). Breslau 1930, S. 211-213; TINTELNOT, H.: Die mittelalterliche Baukunst Schlesiens. In: Quellen und Darstellung zur schlesischen Geschichte, Bd. 1, 1951, S. 116. Wulf Schadendorf gibt davon abweichend eine Errichtungspanne von 1329 bis 1369 an. Siehe SCHADENDORF 1961 (wie Anm. 13), S. 165.

${ }^{26}$ HERMANN - VON WINTERFELD 2015 (wie Anm. 25), S. 626, 679 .

${ }^{27}$ Bei der Chorlösung der Sandkirche sind insofern Ungereimtheiten fassbar, indem die seitlichen Streben der Hauptapside - wenn auch bereits enorm verkürzt - weit in den Bereich vor die Fenster der Seitenchöre hineinragen und diese somit 

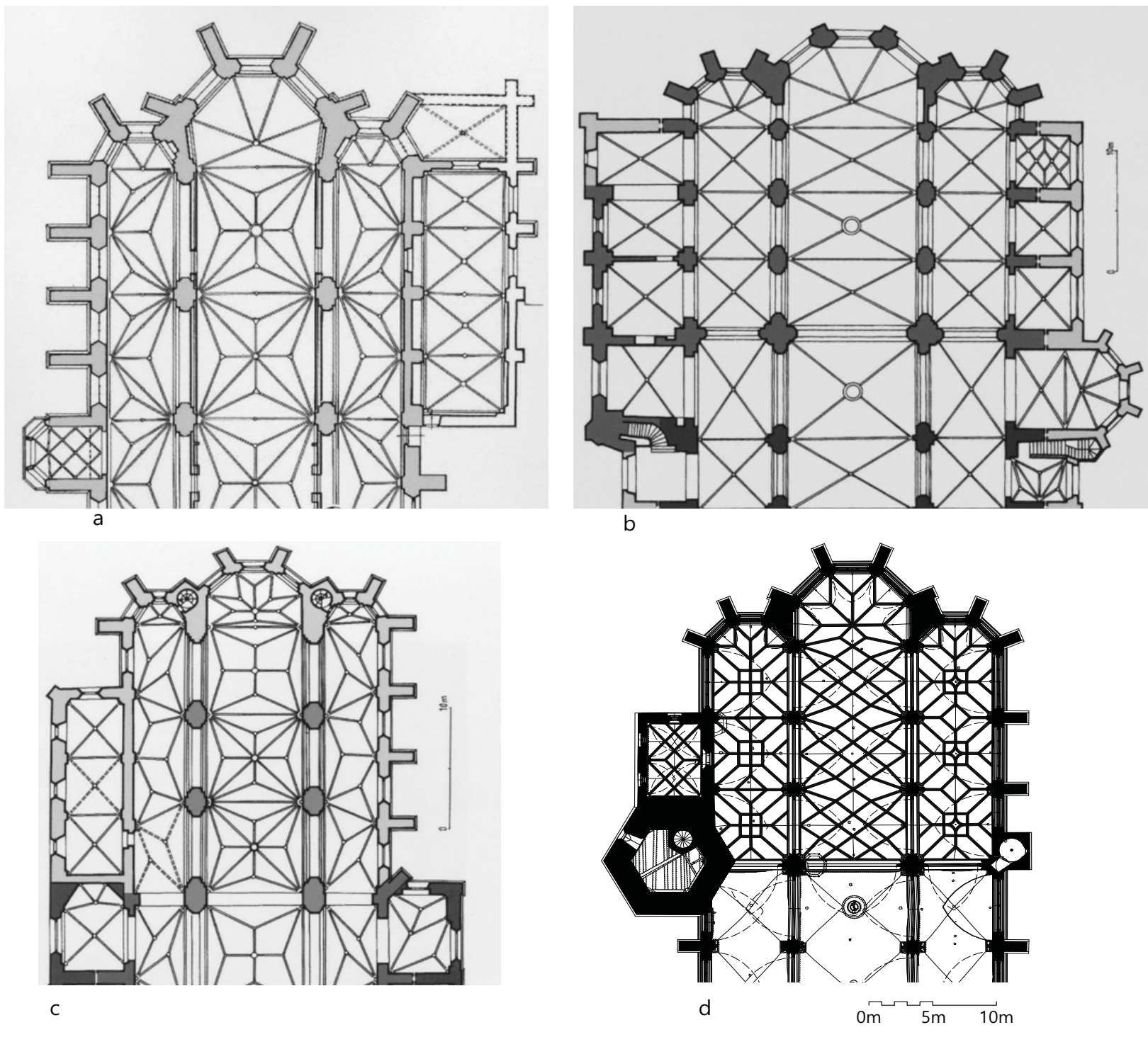

Abb. 9: Vergleich der Grundrisse: Sandkirche in Breslau (a), Elisabethkirche in Breslau (b), Pfarrkirche in Namslau (c), Pfarrkirche in Steyr (d). Zeichnungen 9/ a-c: Mittelalterliche Architektur in Polen. Romanische und gotische Baukunst zwischen Oder und Weichsel, Bd. 2. Eds.: Ch. HERMANN - D. VON WINTERFELD. Petersburg 2015 (überarbeitete Version der bei Architektura gotycka w Polsce, ed. von A. WEODAREK. Warszawa 1995 publizierten Pläne); Zeichnung 9/d: Messbildstelle Dresden

handelt es sich um ein konstruktives Zusammenspiel, das sich zur Standardlösung der im 14. und 15. Jahrhundert in Schlesien bevorzugten Staffelchöre entwickelte und sämtliche der nachfolgenden An-

weitestgehend verdecken. Grund dafür war die räumliche Anpassung des Mittelchors an die seitlichen Polygone. Dazu siehe: Ibidem, S. 679. lagen des schlesischen Raumes - wie beispielsweise die Pfarrkirchen in Namslau (Namysłów, ab ca. 1370/80; Abb. 9/c) und Löwenberg (Lwówek Śląski, 1490-1520) - kennzeichnet. ${ }^{28}$ Außerhalb Schlesiens

${ }^{28}$ Zu den schlesischen Staffelchören (Halle oder Basilika), deren Charakteristika und Verbreitung siehe: Ibidem, S. 636-647, 652-655, 679 . 


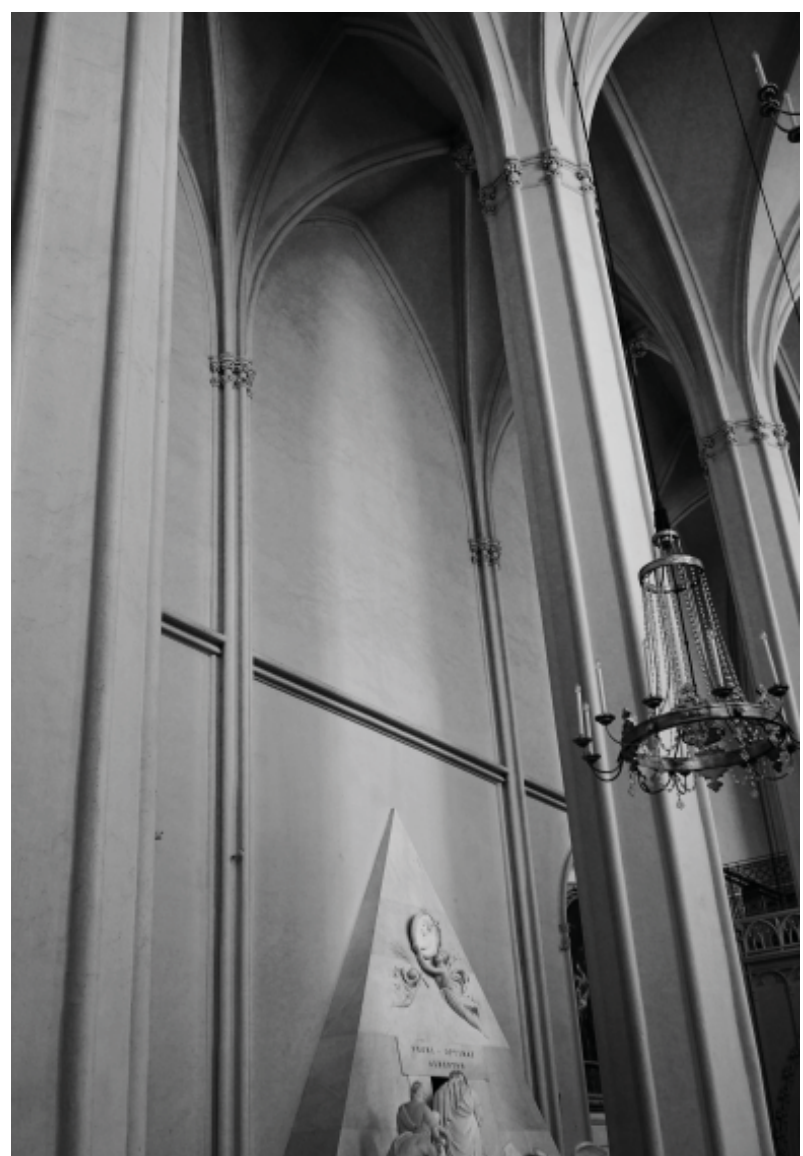

Abb. 10: Wien, Augustinerkirche, Langhaus. Foto: Sophie Morawitz.

werden verwandte Konzeptionen zudem im 15. und beginnenden 16. Jahrhundert in den habsburgischen Erblanden (Steyr [Abb. 9/c], ${ }^{29} \mathrm{Kilb}^{30}$ ), auf dem Ge-

${ }^{29}$ St. Ägidius und Koloman, Chor, ab ca. 1440. Siehe KOCH 1993 (wie Anm. 24), S. 26-27; BÖKER 2003 (wie Anm. 14), S. 214.

${ }^{30}$ Pfarrkirche hl. Simon und Judas, ab 1470. Siehe DEHIO 2003 (wie Anm. 17), S. 929; KOCH 1993 (wie Anm. 24), S. 41; BUCHOWIECKI, W.: Die gotischen Kirchen Österreichs. Wien 1952, S. 285.

31 Kilianskirche, ab 1481. Siehe KOEPF, H.: Die Heilbronner Kilianskirche und ihre Meister (= Veröffentlichungen des Archivs der Stadt Heilbronn, 6). Heilbronn 1961, S. 25.

32 St. Peter und Paul, ab 1423. Siehe DEHIO, G.: Handbuch der Deutschen Kunstdenkmäler. Sachsen I: Regierungsbezirk Dresden. Eds.: GALL, E. et al. München - Berlin 1996, S. 371-373. biet des heutigen Württemberg (Heilbronn ${ }^{31}$ ) sowie im obersächsischen und nordwestböhmischen Raum (Görlitz ${ }^{32}$, Annaberg ${ }^{33}$ ) greifbar.

Übertragen auf Olmütz bedeutet dies, dass das System der Umfassungsmauern bereits ab etwa 1340 als gestalterische Option in Mitteleuropa zur Verfügung stand. Ruft man sich die formale Ausführung der westlichen Abschnitte ins Gedächtnis, fällt es sogar schwer, den Beginn der Arbeiten nicht in die direkte Folgezeit der Breslauer Bauten zu setzen. Immerhin kennzeichnen querrechteckige Seitenschiffjoche (und wohl geplante gleichbreite, wenn auch ausgiebigere, Mittelschiffjoche) auch die Architekturen in St. Elisabeth in Breslau und St. Peter und Paul in Namslau; wobei besonders die Namslauer Hochschiffwände durch die Anzahl und Proportionierung der Joche mit jenen in St. Moritz eng verwandt sind. ${ }^{34}$ Für die nördliche der drei Chorapsiden gilt das Gleiche: Ihre schmale Dimensionierung wirkt, als könnte sie direkt aus Namslau übernommen worden sein und lässt dementsprechend die Interpretation einer - mehr oder weniger - parallel zu den seitlichen Hochschiffwänden verlaufenden Ausführung zu. Selbst die im Mauerzwickel eingefügte Wendeltreppe und der übereckgestellte Strebepfeiler im Übergang zum westlich anschließenden Joch müssen letztlich als Varianten erkannt werden, welche bereits bei den frühen schlesischen Staffelchören anzutreffen sind. ${ }^{35}$

Vergleicht man den bisher besprochenen Befund der Umfassungsmauern mit jenem der Mittel- und Südseitenschiffapsiden, so verdeutlicht sich aber auch, dass letztere zwar grundsätzlich demselben

33 Annakirche, ab 1499. Siehe NUSSBAUM 1994 (wie Anm. 6), S. 306-307, 400 (Anm. 704); DEHIO 1996 (wie Anm. 32), S. 371.

${ }^{34}$ Die Namslauer Pfarrkirche erweist sich auch aufgrund der Tatsache, dass die Freipfeiler erst zu Beginn des 15. Jahrhunderts nach einem abweichenden Jochsystem errichtet worden sind, als äußerst passender Vergleich. Siehe HERMANN VON WINTERFELD 2015 (wie Anm. 25), S. 652-654.

${ }^{35}$ In die Mauerzwickel eingefügte Wendeltreppen finden sich im 14. Jahrhundert bei zahlreichen schlesischen Staffelchören (Halle oder Basilika), so auch in Glatz (Kłodzko, Ostteile vor 1364), Liegnitz (Legnic, 1333-1390), Schweidnitz (Świdnica, 1330 bis E. 15. Jhdt.) Namslau und Striegau (Strzegom, ca. 1360-1391). Bei den bereits angeführten, wenn auch basilika- 

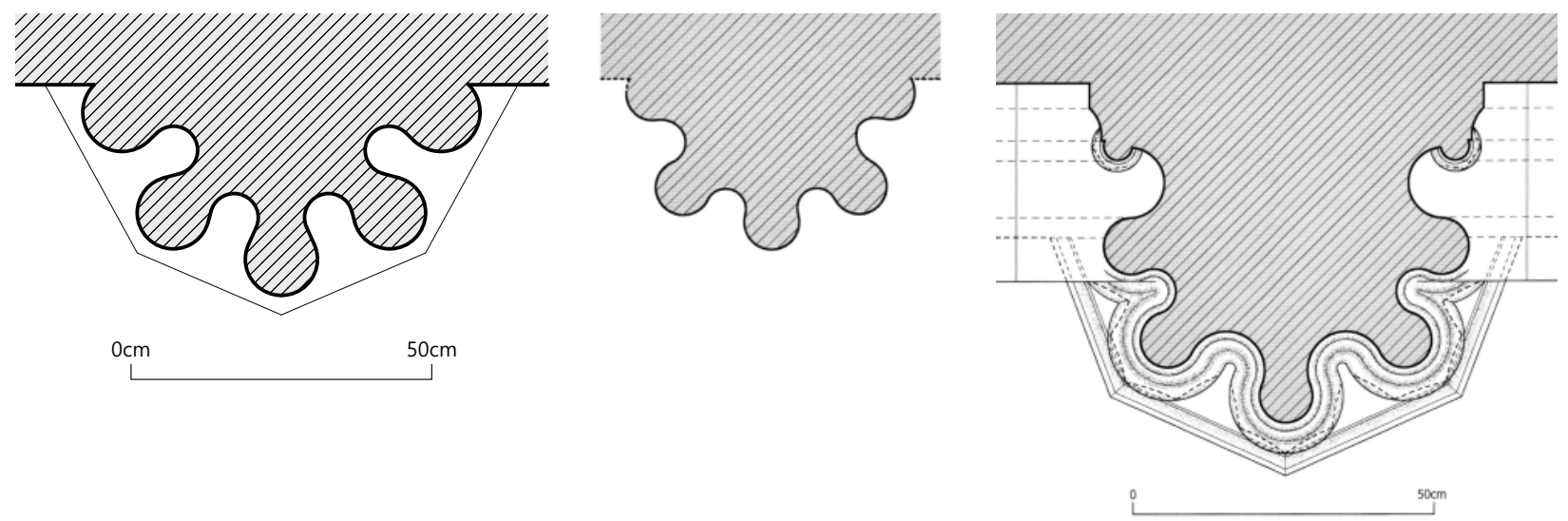

Abb. 11: Maßstabgerechter Vergleich der Vorlagen: Wien, Langhaus Augustinerkirche (a) / Heiligenkreuz, Bernardikapelle (b) / Enns, Wallseerkapelle (c). Zeichnung 11/a: Jakob Kremsner; Zeichnungen 11/b-c: SCHURR, M. C.: Gotische Architektur im mittleren Europa 1220-1340. Von Metz bis Wien. München 2007

Bautyp wie in Namslau oder Breslau entsprechen, ihre Ausführung - rein formal betrachtet - aber erst ab dem 2. Viertel des 15. Jahrhunderts an Wahrscheinlichkeit gewinnt. Die weitreichend aneinander angeglichenen Abmessungen der beiden südlichen Chorschlüsse und das dadurch erzeugte Schiffsverhältnis, wird im Kontext der mitteleuropäischen Staffelchöre erstmals mit dem Bau der Steyrer Stadtpfarrkirche ab den 1440er Jahren (Abb. 7/c) fassbar. ${ }^{36}$ In Folge prägte es sämtliche der dreiapsidialen Hallenchöre der 2. Hälfte des 15. und beginnenden 16. Jahrhunderts (Oppeln [Opole], Löwenberg [Lwówek Śląski], Kilb und Annaberg). Wenngleich der typologische Befund den Schluss nahelegt, die südlichen Chorschlüsse seien erst einige Zeit nach dem Nordpolygon gemäß moderneren Maßstäben errichtet worden, muss eingeräumt werden, dass wir gar nicht wissen, ob - und wenn ja, wie viel - die mittelalterliche Bebauung des Kirchenareals zu der heute fassbaren Situation beigetragen hat. Vor allem

len, Architekturen in Liegnitz und Striegau, finden sich zudem auch bereits übereckgestellte Streben. Zu den Beispielen, deren Eigenschaften und Datierung siehe Ibidem, S. 637-648, 671-672. Zu den Charakteristika schlesischer Staffelchöre im Allgemeinen siehe oben, Anm. 28.

${ }^{36}$ Zur Datierung der Steyrer Stadtpfarrkirche siehe Anm. 29.

${ }^{37}$ Im Aufriss wird das anhand der ausgeführten Maßwerkfenster besonders gut ersichtlich: Während im Nordchor zweibah- wenn man bedenkt, dass das südliche Schiff nicht nur um $0,81 \mathrm{~m}$ breiter als das nördliche Pedant ist, ${ }^{37}$ sondern auch von Westen nach Osten an Ausdehnung zunimmt $(7,71-8,11 \mathrm{~m})$, wird die Frage nach der Einbindung in eine bereits bestehende Infrastruktur unumgänglich - ist laut aktuellem Wissensstand aber nicht zu beantworten. ${ }^{38}$ Dementsprechend muss auch die Auslegung der beobachteten „Ungereimtheiten" offen bleiben. Eines ist aber sicher: Eine Zäsur in der Errichtung ist in beiden Fällen auszuschließen. Der Befund verweist vielmehr auf einen kontinuierlich fortschreitender Ausbau, im Zuge dessen es im Verlauf des ausgehenden 14. und beginnenden 15. Jahrhunderts allenfalls zu formalen Aktualisierungen des Entwurfes kam.

Die Suche nach dem Stil, der die Wandvorlagen prägt, führt in dieselbe Zeit, interessanterweise aber nicht nach Schlesien, sondern in die habsburgischen Kernlande. Hier sind es vor allem die zwischen 1330 und dem ausgehenden 14. Jahrhundert errichteten

nige Fenster sitzen, fanden im Südchor sogar dreibahnige Fensterplatz.

${ }^{38}$ Die Frage nach der mittelalterlichen Bebauung des Moritzfriedhofes und dessen Auswirkung auf den Bau könnte lediglich auf Basis archäologischer Grabungsergebnisse und einer umfassenden Aufarbeitung der historischen Quellentexte erarbeitet werden. Alle angegebenen Maße wurden dem Grundrissplan der NPÚ entnommen und durch eigene Messungen kontrolliert. Zum Plan siehe oben, Anm. 1. 
Sakralräume des Augustinerklosters in Wien, welche durch die Organisation ihrer Dienstsysteme eine auffällige Parallele zeigen (Abb. 10-11/a). ${ }^{39}$ Die aus fünf bzw. vier durch entsprechende Kehlen zusammengefassten Rundstäben gleicher Dimension bestehenden Bündel, die mit der Mauermasse verschmelzenden wandseitigen Dienste und die schlanke Ausbildung der Profile - all das haben die Klosterkirche sowie Ritterkapelle (auch Georgskapelle) der Augustiner in Wien und St. Moritz in Olmütz gemeinsam. In entsprechender Form begegnet uns dieselbe Kombination bereits in der Bernardikapelle der Abteikirche von Heiligenkreuz (1295 geweiht; Abb. 11/b) ${ }^{40}$ sowie der Wallseerkapelle der Franziskanerkirche in Enns

\footnotetext{
${ }^{39}$ Die Errichtungszeiten der einzelnen Abschnitte sind auf Basis der jüngsten Aufarbeitung der Wiener Augustinerkirche und Georgskapelle durch Günther Buchinger und Doris Schön folgendermaßen zu setzen: Kirchenlanghaus, etwa 1330 bis in die 2. Hälfte bzw. 3. Viertel des 14. Jahrhunderts; Chor, ab etwa 1350 bis 1461, wobei die Umfassungsmauer noch im 14. Jahrhundert aufgemauert wurden und lediglich die Einwölbung sowie das Einsetzen der Maßwerke erst um die Mitte des 15. Jahrhunderts realisiert werden konnte; Ritterkapelle (Georgskapelle), etwa 1330er bis 1395. Siehe BUCHINGER, G. - SCHÖN, D.: „,...jene, die ihre hände hilfreich zun bau erheben ...", Zur zeitlichen Konkordanz von Weihe und Bauvollendung am Beispiel der Wiener Augustinerkirche und Georgskapelle. In: RIHA Journal, 2011, 20, Online-Ressource: www.riha-journal.org, Abs. 21-59, 60-89, 113-153.
}

${ }^{40}$ Es muss jedoch betont werden, dass es sich bei der Bernardikapelle um einen weitgehend rekonstruierten Bau handelt. Die Kapelle wurde nach der türkischen Belagerung 1683 verwüstet, anschließend barockisiert und auf Basis der 1910 entdeckten Originalbeständen in den heutigen Zustand gesetzt. Zur Datierung und Baugeschichte siehe: THOME, M.: Kirche und Klosteranlage der Zisterzienserabtei Heiligenkreuz. Die Bauteile des 12. und 13. Jahrhunderts. Petersberg 2007, S. 220 230; SCHURR, M. C.: Gotische Architektur im mittleren Europa 1220-1340. Von Metz bis Wien. München 2007, S. 311.

${ }^{41}$ Für den Hinweis auf Enns bedanke ich mich bei Günther Buchinger. Zur Datierung der Ennser Kapelle siehe: DONIN, R. K.: Die Bettelordenskirchen in Österreich. Zur Entwicklungsgeschichte der österreichischen Gotik (Forschungen zur Landeskunde von Niederösterreich, 3). Baden/Wien 1935, S. 191-201; BRUCHER, G.: Gotische Baukunst in Österreich. Salzburg - Wien 1990, S. 109-112; WAGNER-RIEGER, R.: Mittelalterliche Architektur in Österreich. Eds.: ROSENAUER, A. - SCHWARZ, M. St. Pölten - Wien 1988, S. 139.

${ }^{42}$ Im Unterschied zu Olmütz und Wien besitzen die Vorlagen in Graz allerdings äußerst flache, kaum noch als Kehlen zu be- (ehem. Minoritenkirche, 2. V. 14. Jhds.; Abb. 11/c) ${ }^{41}$, in leicht variierter Gestalt aber auch in der Grazer Leechkirche (1293 geweiht $)^{42}$ sowie den Stiftskirchen von St. Lambrecht (1421 geweiht ${ }^{43}$ und Göttweig $\left(1402 / 03\right.$ bis 1439) ${ }^{44}$. Auch wenn sich das Olmützer Dienstsystem um einiges zierlicher als die eben angeführten Lösungen gestaltet, darf dies von den dargelegten Ähnlichkeiten dennoch nicht ablenken. Ganz im Gegenteil verdeutlicht das Beispiel der Katharinenkapelle der Dominikanerinnenkirche in Imbach (2. V. 14. Jhdts.) ${ }^{45}$, dass entsprechend feingliedrige Runddienste mit trennenden Kehlungen in der zeitgleichen österreichischen Bautradition durchaus eingesetzt wurden. ${ }^{46}$

zeichnende, Zwischenräume. Zur Datierung siehe: SCHURR 2007 (Wie Anm. 40), S. 149, 306; BUCHOWIECKI 1952 (wie Anm. 30), S. 225.

${ }^{43}$ Die St. Lambrechter Lösung unterscheidet sich von jenen in Olmütz und Wien aufgrund deutlich zurückgenommener Kehlungen. Zur Datierung siehe: DEHIO-HANDBUCH: Die Kunstdenkmäler Österreichs. Steiermark (ohne Graz). Wien 2006, S. 447.

${ }^{44}$ In Göttweig wird der mittlere Dienst zusätzlich durch abgesetzte Kanten betont. Zur Datierung siehe: DEHIO 2003 (wie Anm. 17), S. 567.

${ }^{45}$ Die Datierung der Katharinenkapelle betreffend, ist zu erwähnen, dass Richard K. Donin noch von einer Fertigstellung der Kapelle um 1300 ausging. Diese Frühdatierung wurde aber bereits von Renate Wagner-Rieger bezweifelt und eine Errichtung im 2. Viertel des 14. Jahrhunderts erwogen. Dazu siehe: DONIN 1935 (wie Anm. 41), S. 170-177; WAGNER-RIEGER, R.: Gotische Kapellen in Niederösterreich. In: Festschrift für Karl Maria Swoboda. Wien/Wiesbaden 1959, S. 273-307; WAGNER-RIEGER 1988 (wie Anm. 41), S. 86.

${ }^{46}$ Als eine Vorstufe, welche die Entwicklung hin zu den in Olmütz verwirklichten Vorlagen weiter beleuchten könnte, sei auch das Dienstsystem des Chorbaus des 1269 gegründeten Klosters in Imbach erwähnt. Hier finden sich vereinzelt Vorlagen, die sich aus drei Runddiensten mit vermittelnden Kehlen zusammensetzen und von Jiř́ Kuthan und darauf aufbauend auch von Markus Thome mit der bereits angeführten Lösung der Heiligenkreuzer Bernhardikapelle, aber auch den Vorlagen in der um 1260/70 durch Přemysl Otakar (dt. Ottokar) II. errichteten Kapelle der Burg Klingenberg (Zvíkov), in Bezug gesetzt wurden. Auch der Chor der Pfarrkirche in Marchegg (ab 1270er) besitzt einen damit verwandten Aufbau. Zu den österreichisch-böhmischen Vergleichen siehe: THOME 2007 (wie Anm. 40), S. 226-229; KUTHAN, J.: Premysl Ottokar II. König, Bauberr und Mäzen. Höfische Kunst im 13. Jahrhundert. 


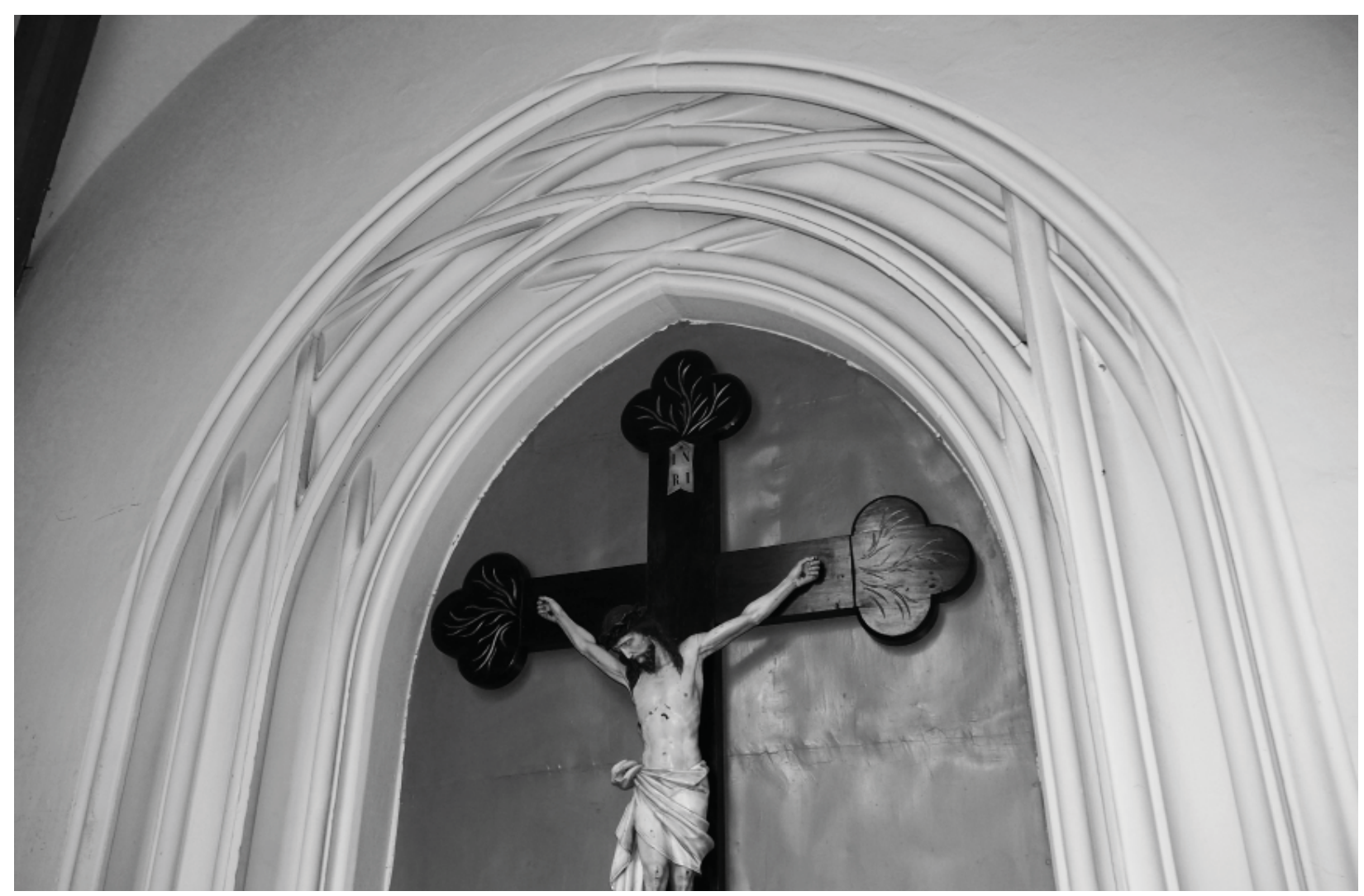

Abb. 12: Olmütz, (Olomouc), St. Mority, Chor, nordöstliches Seitenschiffportal. Foto: Sophie Morawitz,

Der Vergleich mit Imbach überzeugt vor allem dann, wenn man darauf verweist, dass die Kapellen in Imbach, Wien und Enns mit den Wallseer - einem

Wien - Köln - Weimar 1996, S. 201; BUCHOWIECKI 1952 (wie Anm. 30), S. 223.

${ }^{47}$ Eine von Rudolf den Jungen von Liechtenstein im Jahre 1342 getätigte Stiftung verweist im Falle der Wiener Ritterkapelle darauf, dass es sich hierbei um eine Gemeinschaftsstiftung von Herzog Otto den Fröhlichen und seinen Gefolgsleuten (darunter auch die Wallseer, für welche von 1337 und 1341 zusätzliche Stiftungen greifbar sind) handelte. Auch für die Kapelle in Enns ist eine Überlieferung von 1343 greifbar, die den Schluss nahelegt, dass die Kapelle mit der Intention einer Grablege von den Brüdern Reinprecht und Friedrich von Wallsee errichtet worden war. In Falle von Imbach ist zwar keine Stiftung überliefert, doch in Übereinstimmung mit der in der jüngeren Forschung vorgeschlagenen Datierung in das 2. Viertel des 14. Jahrhunderts (dazu siehe Anm. 45) könnte die Errichtung der Kapelle auf eine Stiftung durch Weikard von Winkl, dem Sohn von Ortlieb von Winkl und aus Schwaben stammenden und den habsburgischen Herrschern nahestehendem Adelsgeschlecht - in direkten Zusammenhang zu bringen sind. ${ }^{47}$ Folgt

Gisela, einer Tochter des Imbacher Klosterstifters Albero von Feldsberg, und seiner Gattin Katharina von Wallsee Drosendorf zurückgehen. Dass die Imbacher Katharinenkapelle als Grablege für Mitglieder der Familie Wallsee-Drosendorf gedient haben könnte, vermutete bereits Renate Wagner-Rieger - argumentierte aber über die Schwester Katharinas, Anna von Wallsee, welche 1332 als Nonne im Imbacher Kloster erscheint. Auch Marc Carel Schurr bezeichnet die Imbacher Kapelle als Stiftung der Wallseer, ohne jedoch näher darauf einzugehen. Zu Wien siehe: BUCHINGER - SCHÖN 2011 (wie Anm. 39), Abs. 60-61. Zu Enns siehe: HAGENEDER, H.: Beiträge zur Geschichte der Minoriten in Enns von den Anfängen bis 1553. In: Mitteilungen des Oberösterreichischen Landesarchivs, 11, 1974, S. 254. Zu Imbach siehe: BUCHINGER, G.: Die Katharinenkapelle. In: Das ehemalige Dominikanerinnenkloster in Imbach. Geschichte - Architektur - Bauforschung. St. Pölten 2020, Artikel in Vorbereitung; WAGNER-RIEGER 1959 (wie Anm. 45), S. 293; SCHURR, M. C.: Stilpluralismus, 
man Marc-Carel Schurr, so könnte sich in Übereinschau mit den Pfeilern des Chors von St. Stephan in Wien - welchen eine verwandte Gestaltungsweise mit runden Diensten zu eigen ist - sogar eine Art Hofstil unter den Habsburgern und deren Umfeld abzeichnen. ${ }^{48}$ Mag sein, dass sich über die Wiener Pfeiler wiederum ein Bogen zur Oppenheimer Katharinenkirche (Langhaus, 1317 bis ca. 1340) schlagen lässt, die Schurr als direktes Vorbild anführte und im westlichen Abschnitt Vorlagen ähnlicher Ausformung besitzt. ${ }^{49}$

Kommt man diesen Beobachtungen nach, ergibt sich für die Chorumfassungsmauern der Olmützer Moritzkirche also keineswegs dasselbe Resultat wie für die weitgespannten Freipfeiler. Vielmehr müssen ihnen Merkmale zuerkannt werden, die zwischen den 1330/40er Jahren und dem 1. Drittel des 15. Jahrhunderts im östlichen Mitteleuropa zu beobachten sind. Dass der Hallenchor im ausgehenden 14. Jahrhundert tatsächlich schon im Bau war, lässt sich nicht zuletzt einer bisher kaum berücksichtigten Urkunde

Stilentwicklung oder Hofstil? Gedanken zu Formphänomenen am Chor der Wiener Stephanskirche. In: Wiener Jabrbuch für Kunstgeschichte, 62, 2014, S. 27-28. Zu der Familie Wallsee im Allgemeinen siehe: HRUZA, K.: Die Herren von Wallsee. Geschichte eines schwäbisch-österreichischen Adelsgeschlechts (1171-1331) (= Forschungen zur Geschichte Oberösterreichs, 18). Linz 1995; DOBLINGER, M.: Die Herren von Walsee. Ein Beitrag zur österreichischen Adelsgeschichte. In: Archiv für österreichische Geschichte, 95, 1906, S. 235-578.

${ }^{48}$ SCHURR 2014 (wie Anm. 40), S. 26-28.

${ }^{49}$ In Oppenheim kann Rudolf von Habsburg zumindest als Stifter eines Teils der Chorverglasung gefasst werden. Siehe Ibidem, S. 26-27. Zur Datierung siehe: SCHÜTZ, B.: Die Katharinenkirche in Oppenheim. Berlin/New York 1982, S. $177-217$.

${ }^{50}$ Der originale Quellentext lautet in den, für den Kirchenbau, relevanten Abschnitten folgendermaßen: erigendum ac in honore sancte Anne, matris virginis Marie, et sancti Augustini consecrandum in nova capella in cimiterio cum corpore ecclesie sancti Mauricii prope turrim novam inchoata. Zudem ist anzuführen, dass August Prokop angibt, dass zwischen 1357 und 1362 Zuwendungen in Form von Spenden greifbar sind, von denen eine auch explizit auf ein Bauvorhaben hinweist: „,[...] der Stadtrat widmet nämlich 23 Mark zur Vergrößerung des Schiffes [...]“. Da diese Überlieferungen in den Werken anderer Autor*innen keine Erwähnung finden und auch nicht nachvollzogen werden kann, auf welche Quellenangaben sich Prokop in seiner aus dem Jahre 1403 entnehmen. In dieser heißt es, dass die Errichtung der Kapelle der hl. Anna und des hl. Augustin am Moritzfriedhof zeitgleich mit Arbeiten am neuen Kirchenbau von statten ging. ${ }^{50}$ Auch wenn im Quellentext lediglich von corpore ecclesie sancti Mauricii die Rede ist, kann auf Basis der Tatsache, dass für den Bau des Langhauses zwischen 1419 und 1452 eine Reihe von Stiftungen greifbar ist,$^{51}$ dennoch eine Benennung der Chorsubstanz quasi vorausgesetzt werden.

So gesehen muss nicht nur die Errichtung des gesamten Ostabschnittes im Verlauf der 2. Hälfte des 15. Jahrhunderts, sondern auch der tradierte Baubeginn des neuen Kirchenbaues im Jahre 1412 (Bauinschrift Nordwestturm), ${ }^{52}$ gründlich revidiert werden. Mehr noch: Letztlich entpuppt sich der Chorbau als fulminanter Auftakt des spätmittelalterlichen Sakralbauprojektes und ,[...] die Baugeschichte der Kirche landet vom Kopf auf den Füßen. ${ }^{\text {"53 }}$ Für die Prokop'sche Bauchronologie bedeutet dies, dass sie als zutreffend angesehen werden muss. Es ist jedoch

Aussage stützt, kann vorerst nicht auf Basis dieser argumentiert werden. Es muss natürlich auch in Erwägung gezogen werden, dass Prokop sich auf Dokumente stütze, die heute eventuell gar nicht mehr greifbar sind. Zur Überlieferung sowie der Kapelle der hl. Anna und des hl. Augustin siehe BISTŘICKÝ, J.: Dvě poznámky ke středověkému místopisu Olomouce. In: Vlastivědný věstník moravský, 29, 1977, S. 63. Zu den Angaben von Prokop siehe: PROKOP 1904 (wie Anm. 1), S. 428.

51 Überlieferungen die sich eindeutig auf den Westabschnitt von St. Moritz (Nordwestturm und Langhaus) beziehen lassen: 1435, Kapellenstiftung im Nordwestturm; 1440, Stiftung für den Altar des hl. Kreuzes beim Pfeiler des neuen Turms; 1443, Gesuch um Konfirmation des Altars der hll. Drei Könige circa unam columnam contra turrim antiquam; 1449, Stiftung für den Altar der hl. Dreifaltigkeit im neuen Turm. Zu den Stiftungen siehe: HLOBIL - TOGNER - HYHLÍK 1992 (wie Anm. 2), S. 5; KUX 1937 (siehe Anm. 21), S. 107-108; WOLNY 1946 (wie Anm. 9), S. 75; WOLNY 1855 (wie Anm. 9), S. 226; PROKOP 1904 (wie Anm. 1), S. 429; NOWAK 1890 (wie Anm. 1), S. 2-3.

${ }^{52}$ Siehe NOWAK 1890 (wie Anm. 1), S. 2; BENEŠOVSKÁ 2001 (wie Anm. 2), HLOBIL - TOGNER - HYHLÍK 1992 (wie Anm. 2), S. 3-4; BLÁHOVÁ 1999 (wie Anm. 2), S. 204; RYDLOVÁ 2014 (wie Anm. 7), S. 16.

${ }^{53}$ Zitat von Michael Viktor Schwarz aus dem Gutachten der Masterarbeit. 
einzuräumen, dass die Anerkennung der von Prokop gelieferten Ergebnisse, nicht gleichermaßen für seine Beweisführung gilt: So begegnen uns in seinem Kapitel zu St. Moritz diverse unzureichend belegte Quellen und eine - in Anbetracht der oben gelieferten Vergleiche - fragwürdig erscheinende typologische Ableitung von den Grundrisskonzeptionen der Teynkirche und Emmauserstiftskirche in Prag (Prague); eine Stilanalyse fehlt zudem vollkommen. ${ }^{54}$ Erst durch die hier unternommenen Verifizierungen, für welche vor allem die Anfertigung eines Grundrisses nach Naturmaß grundlegend war, konnte Prokops Frühdatierung an Evidenz gewinnen.

Folgt man dieser, verdeutlicht sich aber auch, dass bis zur Fertigstellung noch etliche Jahrzehnte verstrichen sind. Immerhin hatte die Analyse der reichprofilierten Bündelpfeiler (Abb. 4-5) zum Ergebnis, dass der Innenausbau zum bestehenden Hallenchor erst im Verlauf der 2. Hälfte des 15. Jahrhunderts einsetzte. Selbst die Laubblattkapitelle (sowohl jene der Stützen als auch jene der Umfassungsmauern) und engmaschigen Netzfigurationen (Abb. 1-2) ordnen sich ebenso wie die Mehrzahl der Maßwerkentwürfe einer Formensprache unter, die im ausgehenden Mittelalter neu aufkam. Die am westlichsten Schlussstein des Mittelschiffgewölbes inschriftlich angebrachte Jahreszahl 1483 muss des Weiteren als Beweis für eine weitreichende Vollendung der Bauarbeiten in den letzten Jahrzehnten des 15. Jahrhunderts gesehen werden. Eine Ausnahme bildeten die seitlichen Eingangsportale (Abb. 12), deren virtuos verstrebten Profilierung enge Übereinstimmungen zu den Portalen an der Brünner Jakobskirche (nach 1480; Abb. 13) ${ }^{55}$ und dem Nordportal der Brünner Domkirche (1490-1530) ${ }^{56}$ zeigen.

${ }^{54}$ Zu Prokops Ausführungen siehe PROKOP 1904 (wie Anm. 1), S. 428-432. Zu den Prager Sakralbauten und deren Datierung siehe unten, Anm. 64 und 65.

${ }^{55}$ Jan Sedlák nimmt an, dass die Bauarbeiten am Chor der Jakobskirche erst nach 1480 wieder aufgenommen wurden. Dementgegen setzte Jaroslav Bureš die Ausbauphase zur heutigen Hallenkirche bereits in die Zeit zwischen etwa 14561473. Je nachdem zu welchem Ergebnis man kommt, fällt die Errichtung des nordöstlichen Seitenschiffportals in das 3. Viertel des 15. Jahrhunderts oder in die Zeit zwischen 1480 und den ersten Jahrzehnten des 15. Jahrhunderts. Die beiden westlichen Portale werden einstimmig in die ersten Jahrzehnte des 16. Jahrhunderts gesetzt. Zu den Thesen von Sedlák und

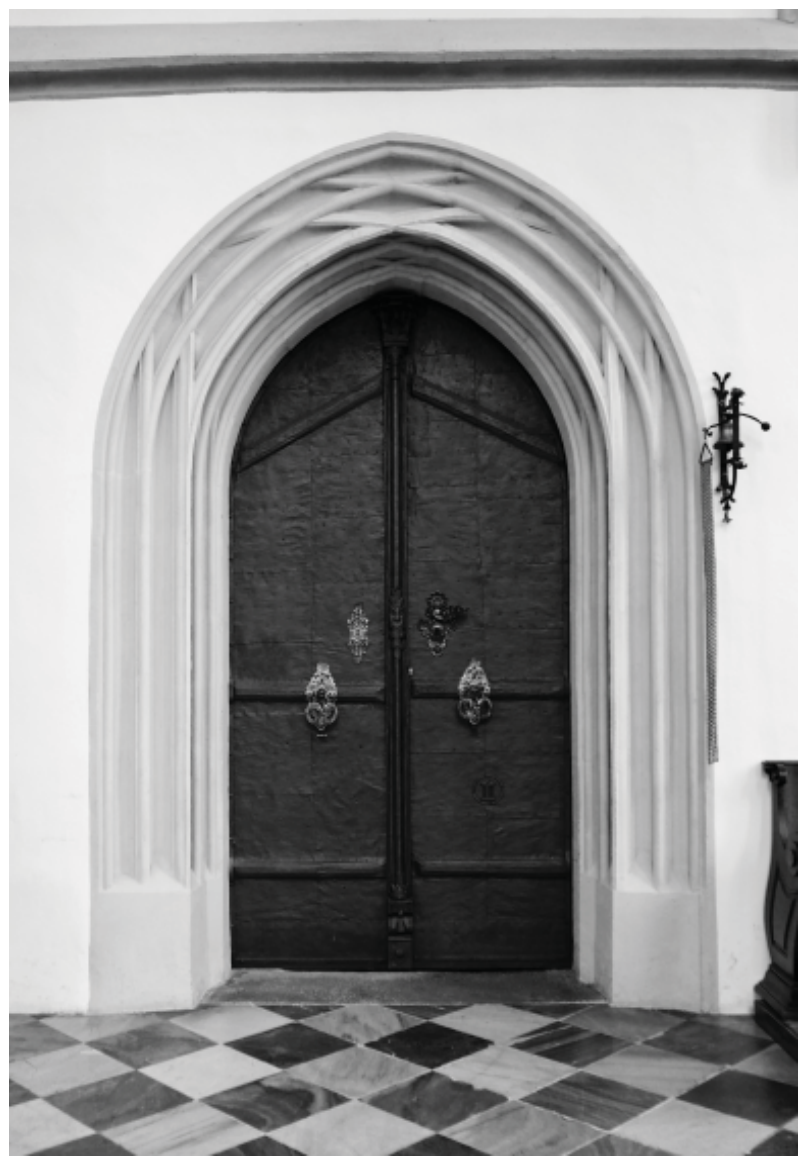

Abb. 13: Brünn (Brno), St. Jakob, Portal in der nördlichen Hochschiffwand des Chors. Foto: Sophie Morawitz.

Die am Südportal angebrachte Miniaturarchitektur in Form von Baldachinen ist zudem mit der südlichen Portalvorhalle der Steyrer Stadtpfarrkirche (um bzw.

Bureš siehe vor allem: SEDLÁK 1971 (wie Anm. 5), S. 143; BUREŠ 1972 (wie Anm. 18), S. 100. Siehe auch: KROUPA 1999 (wie Anm. 3), S. 94-95; KROUPA - ŠEFERISOVÁ JAKUBEC 2015 (wie Anm. 3), S. 264-265. Zur Datierung der westlichen Abschnitte siehe: KROUPA 1999 (wie Anm. 3), S. 95-96; KROUPA - ŠEFERISOVÁ - JAKUBEC 2015 (wie Anm. 3), S. 265; BENEŠOVSKÁ 2001 (wie Anm. 2), S. 265. Zu Literatur über St. Jakob im Allgemeinen siehe auch oben, Anm. 3.

${ }^{56}$ August Prokop datiert den spätmittelalterlichen Ausbau der Brünner Domkirche, und somit auch die Errichtung des gemeinten Portals, in die Zeit zwischen 1490 und 1530. Siehe PROKOP 1904 (wie Anm. 1), S. 575. Die Ähnlichkeiten 
nach 1500$)^{57}$ verwandt. ${ }^{58}$ Demnach ist die Errichtung der Seitenportale in die 1490er bis 1520er Jahre zu setzten, was wiederum bedeutet, dass der Bau des Chors - je nach Ansatz des Baubeginns - mindestens 120 bis 150 Jahre dauerte (ca. 1370/1400-1520).

Von den Hussitenkriegen einmal abgesehen, erklärt sich dieser langsame Fortschritt vor allem durch den Umstand, dass ab 1412 auch am nördlichen Westturm und zwischen etwa 1435 und 1453 am Langhaus gearbeitet wurde. ${ }^{59}$ Nach den Chorumfassungsmauern und Turmuntergeschossen wäre man also direkt an die Errichtung der dazwischenliegenden Langhauswände gegangen und hätte erst nach Vollendung dieser sämtliche Freipfeiler in einem Zuge aufgerichtet sowie die Einbauten des gesamten Sakralbaus realisiert. ${ }^{60}$

zwischen dem Nordportal des Olmützer Hallenchores und der Portalrahmung der Brünner Domkirche, wurde bereits von Zdenka Bláhová erkannt, welche darüber hinaus auch das Portal der Kirche Mariä Himmelfahrt in Wischau (Vyškov) als wichtige Parallele anführt. Siehe BLÁHOVÁ 1999 (wie Anm. 2), S. 205.

${ }^{57}$ Die südliche Portalvorhalle wird von Rudolf Koch in die Amtszeit von Wolfgang Tenk gesetzt, welcher von 1483 bis 1513 als Baumeister in Steyr fassbar ist. Siehe KOCH 1993 (wie Anm. 24), S. 49-50.

${ }^{58}$ Während Zdenka Bláhová die Baudetails des Südportals als Elemente interpretierte, die sich auf die Formensprache der unter Peter Parler entstandenen Portale orientieren, wurden die auffälligen Übereinstimmungen zum Formenrepertoire der Wiener Bauhütte bereits von Hana Myslivečková gebührend herausgestrichen. Myslivečková versuchte die Verbindungen nach Wien sogar mithilfe der am Portal fassbaren Steinmetzzeichen zu belegen, indem sie angibt, dass eines der insgesamt vier vorhandenen Steinmetzzeichen auch am zwischen 1467 und 1513 errichteten Nordturm des Wiener Stephansdomes fassbar ist. Dieses ist in den von Alois Kieslinger zusammengetragenen Katalog an Steinmetzzeichen auf welchen sie sich beruft - aber gar nicht aufgelistet. Auf Nachfrage konnte eruiert werden, dass Myslivečková das bei Kieslinger unter der Nr. 99 (Treppenhaus des Südturm) angeführte Steinmetzzeichen, aber auch das unter der Nr. 207 (Kanzel) aufgelistete Zeichen meint. Beide sind zwar ebenso als $Z$ ausgeformt, ist in ihrer Gestaltung aber keinesfalls mit jenem an der Moritzkirche ident. Für die Weiterleitung dieser Informationen bedanke ich mich bei Hana Myslivečková (NPÚ ÚOP v Olomouci). Ähnlich schwierig gestaltet sich auch die Lage in Bezug auf die ebenfalls am Südportal anzutreffende Jahreszahl 15 - (?) 1. Während diese von Bláhová und Myslivečková als 1521 interpretiert und darauf aufbauend
Bei genauerer Betrachtung lässt sich ein derartiges Rekonstruktionsmodell des Baufortschrittes sogar mithilfe des erhaltenen Primärbefundes untermauern. Die Stützen des Kirchenbaues weisen nämlich nicht nur ein nahezu einheitliches Erscheinungsbild auf, ${ }^{61}$ sondern reihen sich auch in nahezu gleichbreiten Abständen zwischen dem Nordturm und den Chorpolygonen auf (Abb. 2). Letztere mussten beim Aufbau der Langhauspfeiler also bereits bestanden haben. Hinzu kommt, dass sich im Bereich des Dachbodens der sog. alten Sakristei, die im Norden an der Schwelle zwischen Chor und Langhaus angebaut ist, eine Baunaht befindet, deren leichte Abtreppung von links oben nach rechts unten belegt, dass das östliche Mauerwerk des Chors als chronologisch älter als die westliche Struktur der Langhausmauer angesehen werden muss (Abb. 14). ${ }^{62}$

als Errichtungs- bzw. Vollendungsdatum der Portalrahmung angesehen wird, kann diese Auslegung aufgrund der kaum lesbaren dritten Ziffer von links keineswegs bestätigend übernommen werden. Selbst für die vierte Ziffer von links erscheint die Zahl sieben als weitaus zutreffender. Die Inschrift darf also keineswegs als Garant für eine Errichtung im zweiten Jahrzehnt des 16. Jahrhunderts herangezogen werden. Siehe BLÁHOVÁ 1999 (wie Anm. 2), S. 205; MYSLIVEČOVÁ, H.: K výpovědní hodnotě kamenických značek kostela sv. Mořice v Olomouci. In: Kamenické a domovni značky (merky). Sbornik př̀spèvkù ze semináre 1989 v Olomouci. Ed.: MILLER, K. Ostrava 1991, S. 43-44, 49; KIESLINGER, A.: Die Steine von St. Stephan. Ed. vom Verein für Denkmalpflege in Wien. Wien 1949, S. 169-180.

59 Zu den Überlieferungen für die Arbeiten am Westabschnitt der Kirche (Nordwestturm und Langhaus) siehe oben, Anm. 51.

${ }^{60}$ Die von Ivo Hlobil angegebene Dotierung ,[... $]$ zur Herstellung von Fenstern [...]“" aus dem Jahr 1453 könnte folglich für sämtliche Fenstermaßwerke herangezogen werden. Zur Überlieferung siehe: HLOBIL - TOGNER - HYHLÍK 1992 (wie Anm. 2), S. 5.

${ }^{61}$ Die etwas reicher gehaltene Ausformung der östlichen Pfeiler muss dabei als eine bewusste Abhebung des Chorbereiches interpretiert werden.

${ }^{62}$ Diese Beobachtung könnte darüber hinaus auch durch die Tatsache untermauert werden, dass die nördliche Hochschiffwand des Langhauses in ihren westlichsten Abschnitten aus einem - im Vergleich zum östlich daran anschließenden Mauerwerk des Chors - deutlich unordentlicheren Kompartimentmauerwerk zusammensetzt und somit für eine jüngere Entstehung spricht. Problematisch gestaltet sich in Hinsicht auf die Methode der komparativen Mauerwerksanalyse 
Die bisher gemachten Beobachtungen laufen schließlich auf ein Ergebnis hinaus, das über die Baugeschichte hinweg auch Konsequenzen für die kunsthistorische Bewertung des Bauwerks hat. Während die bisherige Spätdatierung den Olmützer Hallenchor zu einem Unterfangen gemacht hat, das in seiner Gesamtheit in die Blütezeit der Bischofsstadt nach den hussitischen Unruhen - und somit einer Zeit des gesteigerten künstlerischen Austausches zwischen Mähren, Böhmen und der Donauregion - fiel, bettet ein Baubeginn vor 1400 das Projekt in eine Entwicklung, deren wichtigste Etappen in Schlesien aber auch in Österreich greifbar werden. So konnte herausgearbeitet werden, dass die in Schlesien entwickelten Staffelchöre wohl die Voraussetzung für die Disposition der Moritzkirche bilden. Eine Mutmaßung, welche schon allein aufgrund der Tatsache, dass sich die Besitztümer des Olmützer Bischofs in der 2. Hälfte des 14. Jahrhunderts bis in das angrenzende Herzogtum hinein ausbreitenden und einen engen Kulturaustausch mit diesem bewirkten, keineswegs abwegig ist. Als relevante Vorläufer für die in Olmütz angewandte Hallenchorlösung mit 5/8-Chor im Mittelschiff und flankierenden 3/8-Schlüssen könnten sich demnach die Architekturen in Breslau und Namslau hervortun und nicht die bisher vorgeschlagenen Entwürfe in Steyr oder Prag. ${ }^{63}$ Entgegen der Steyrer Stadtpfarrkirche - die

jedoch, dass diese in der tschechischen Forschung nicht angewendet wird und folglich kein Chronologiegerüst für den mährischen Raum als Datierungsgrundlage der in St. Moritz befundeten Mauerwerksstrukturen vorliegt. Es darf aber angenommen werden, dass die für den nordostösterreichischen Raum herausgearbeitete historische Entwicklung der Mauerstruktur mit den Bestand in Südmähren in Einklang zu bringen ist (für diesen Hinweis bedanke ich mich bei Günther Buchinger). Dementsprechend ist auch im Falle des nordmährischen Gebietes von einer weitreichend übereinstimmenden relativchronologischen - wenn nicht sogar absolutchronologischen - Entwicklung auszugehen. Zur Methode der komparativen Mauerweksanalyse und dem Chronologiegerüst des nordostösterreichischen Raumes siehe: SEEBACH, G.: Zeitspezifische Strukturen des mittelalterlichen Mauerwerks. In: Burgen und Ruinen. Von Quadern und Mauern, Denkmalpflege in Niederösterreich, 12, 1994, S. 19-23; KÜHTREIBER, K. - KÜHTREIBER T.: Methodische Grundlagen zur archäologischen und bauhistorischen Erfassung von Burgen im Pittener Gebiet. In: KÜHTREIBER, K. et al: Webrbauten und Adelssitze Niederösterreichs. Das Viertel unter dem Wienerwald, Bd. 1. St. Pölten 1998, S. 1-17; MITCHELL, P. - SCHÖN,

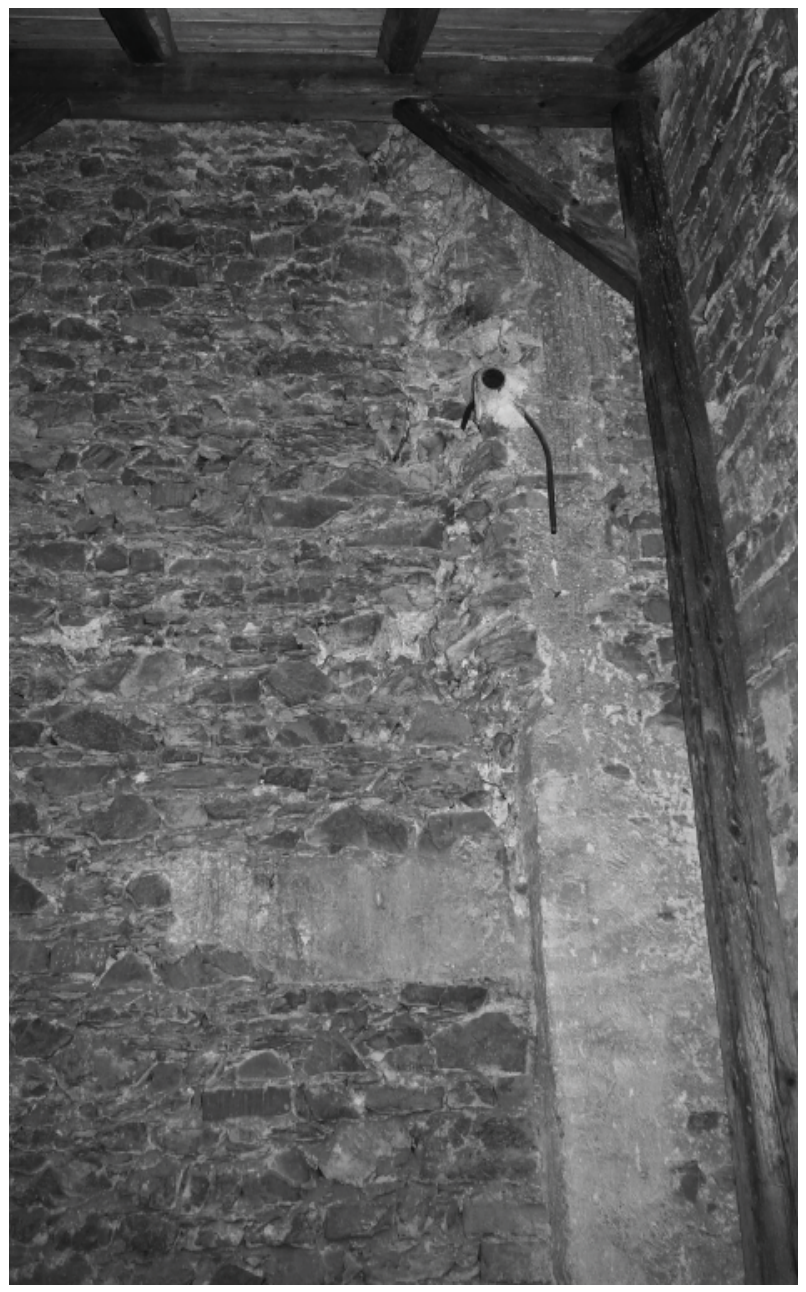

Abb. 14: Olmütz, (Olomouc), St. Mority, Baufuge in der nördlichen Hochschiffwand zwischen Chor (links) und Langhaus (rechts). Foto: Sophie Morawitz.

D.: Struktur und Datierung des Mauerwerks in Wien. In: Österreichische Zeitschrift für Kunst und Denkmalpflege, 56, 2002, S. 462-473.

${ }^{63}$ Auf August Prokop aufbauend, verwiesen auch Dobroslav Líbal und Michaela Rydlová auf die Vorbildfunktion der Teynkirche und Emmausklosterkirche. Ergänzend betonten Walther Buchowiecki, Rudolf Koch, Norbert Nussbaum und Michaela Rydlová Verbindungen zum Chor der Steyrer Stadtpfarrkirche, aber auch dem Albertinischen Chor der Wiener Stephanskirche, der innerhalb der österreichischen Kunstgeschichte als unmittelbares Vorbild der Steyrer Chorlösung gilt. $\mathrm{Zu}$ den typologischen Vergleichen siehe BUCHOWIECKI, W.: Die gotische Hallenkirche in Österreich, Diss. (ms.). Wien 1932, S. 190; BUCHOWIECKI 1952 (wie Anm. 29), S. 457; KOCH 1993 (wie Anm. 24), S. 44; NUSSBAUM 1994 (Wie Anm. 6), 
aufgrund ihrer Errichtung nach 1440 als Richtmaß ausscheidet -, sind die Prager Beispiele der Teynkirche (ca. 1350-1420) ${ }^{64}$ und Emmauserklosterkirche (Na Slovanech, 1347-1372) ${ }^{65}$ zeitlich durchaus im Rahmen. Bei konsequenter Analyse tut man sich allerdings schwer, die hier realisierten Entwürfe als direkte Vorlagen zu verstehen, da diese mit der Olmützer Lösung lediglich aufgrund der Tendenz hin zu einer weitreichenden Assimilierung der Chorpolygone sowie -schiffe übereinstimmen.

Festzuhalten ist ebenso, dass das in der Grundrisskonzeption fassbare Suchen nach innovativen und abseits der lokalen Bautradition entwickelten Gestaltungsprinzipien, auch für die realisierten Architekturdetails eine naheliegende Variante darstellt: Auch wenn die Möglichkeit einer eigenständigen Formfindung im Falle der Olmützer Vorlagengestaltung keineswegs vollkommen ausgeschlossen werden darf, erscheint es in Anbetracht der dargelegten Analogien logischer, diese als Abbilder der österreichischen Lösungen zu interpretieren. Fraglich bleibt jedoch, welche Rolle den Beziehungen der Habsburger, aber auch dem Netzwerk der Wiener Bauhütte (denn der in Wien tätige Ulrich Nußdorfer ist auch für die Bauarbeiten in Göttweig überliefert und im Falle der Wallseer-Stiftungen müssen ebenso Kontakte zu den in Wien tätigen Handwerkern vorausgesetzt werden), hinsichtlich ihrer Entwicklung und Verbreitung tatsächlich zukam und ob das Re-

S. 235; RYDLOVÁ 2014 (wie Anm. 7), S. 19-21; PROKOP 1904 (wie Anm. 1), S. 431-432; LÍBAL, D.: Gotická architektura $v$ Čechách a na Moravě. Prag 1948, S. 161.

${ }^{64}$ Siehe BENEŠOVSKÁ 2001 (wie Anm. 1), S. 94.

${ }^{65}$ Siehe Ibidem, S. 88.

${ }^{66}$ Dazu siehe oben, Anm. 5 und 6.

${ }^{67}$ In den Satzungen des Regensburger Hüttentages von 1459 heißt es: Item Maister Larencz. Spennig werchmaister des paws zu Sand Steffan zu Wien zu gleicher weis als vorgeschriben stet in sein puechssen geben werden im land zu Lambach Steyren Burckhawssen Vungarn ens und die Thunaw abbin öbrister Maister. Eine Abschrift der Satzungen des Regensburger Hüttentages findet sich in dem vom späteren Steyrer Baumeister Wolfgang Tenck im Jahre 1480 verfassten Admonter Hüttenbuch im Johanneum zu Graz. Zum Regensburger Hüttentag und Admonter Hüttenbuch siehe: NEUWIRTH, J.: Die Satzungen des Regensburger Steinmetzentages nach dem Tiroler Hüttenbuche von konstruktionsmodell einer Hofkunst den Gegebenheiten überhaupt gerecht werden kann.

Darüber hinaus löst sich das Bauwerk auch in seiner spätmittelalterlichen Ausbauphase weitgehend aus den tradierten böhmischen Zusammenhängen und ordnet sich baukünstlerischen Errungenschaften der Wiener Hütte unter, die, allem Anschein nach, über Brünn transferiert wurden. Von Sedlák, Hlobil, Bláhová, Nussbaum und Lepsky bereits in seinen Ansätzen herausgearbeitet, ${ }^{66}$ blieb das tatsächliche Ausmaß des dahinterliegenden Bezugsgeflechtes dennoch bis heute unerkannt: Denn augenfällige Parallelen zu den zeitgleichen Wiener aber auch Brünner Lösungen zeichnen sich nicht nur in einigen wenigen Aspekten des Chorbaues ab, sondern prägen sämtliche der in der 2. Hälfte des 15. Jahrhunderts und dem beginnenden 16. Jahrhundert zur Ausführung gekommenen Baudetails. Dabei könnte man insbesondere die figurierten Rautennetze (Abb. 1-2) und antiquierten Freipfeiler (Abb. 4-5) für gelungene Kopien nach Wiener Vorbild halten. Doch das Wirkungsgebiet der Wiener Hütte war mit der wachsenden politischen und kulturellen Stellung Wiens rasant angewachsen und reichte im ausgehenden Mittelalter allen Anschein nach die Thunaw abhin bis nach Ungarn - und wohl auch Mähren -, wodurch auch eine aktive Beteiligung der Wiener Bauhütte am Baugeschehen in Olmütz in Erwägung $\mathrm{zu}$ ziehen ist. ${ }^{67}$ Gleichwohl sei betont, dass beide

1460. Berlin 1896, S. 53; Gotik Schätze Oberösterreich. Eds.: SCHULTES, L. - PROKISCH, B. [Kat. Ausst.]. Linz 2002, S. 231. Dass die Architektur der Wiener Hütte im ausgehenden Spätmittelalter auch tatsächlich bis in das historische Ungarn hinein wegweisend wurde, wird durch einen Brief von Laurenz Spenning (Wiener Dombaumeister 1454/55-1477) an den Pressburger Stadtrat aus dem Jahre 1458 sogar schriftlich belegt. In diesem beruft sich der Wiener Dombaumeister auf zwei aus Preßburg erhaltene Briefe, in welchen vom Pressburger Stadtrat um eine Empfehlung für die Auswahl eines fähigen Fachmanns für die Position des dortigen Hüttenbaumeisters hinsichtlich der Arbeiten am Chor der Martinskirche (1459-1478) gebeten wird. Spenning kam folglich zumindest eine wichtige beratende Funktion zu. Es muss jedoch erwähnt werden, dass Bureš sogar vermutet, dass Laurenz Spenning den Bauarbeiten als eine Art planender Architekt im neuzeitlichen Sinne überstand. Ähnliches zieht Petr Kroupa auch für die Arbeiten am Chor der Brünner Jakobskirche in Betracht. Dazu siehe: BUREŠ 1972 (Wie Anm. 18), S. 85, 95; BÖKER 2003 (wie Anm. 14), S. 222; KROUPA 1999 (wie Anm. 3), S. 95. Zu Laurenz Spenning siehe: PERGER, R.: Baumeister des 
Denkmodelle, einen engen Austausch der in Olmütz, Brünn und Wien tätigen Handwerker voraussetzen und einmal mehr herausstreichen, dass der Chor der Moritzkirche, wie er sich heute präsentiert, ohne den internationaleren Formentransfer des ausgehenden Mittelalters nicht denkbar wäre.

Abschließend sei zusammengefasst, dass sich der Hallenchor von St. Moritz in Olmütz nunmehr anders präsentiert als zuvor: Als anspruchsvolle Bauetappe, die keineswegs als ,[...] next stage $[\ldots]^{668}$ nach der Vollendung von Nordwestturm und Langhaus abgetan werden will, sondern als Startschuss des spätmittelalterlichen Neubauprojektes. Begonnen wurde dabei mit den Umfassungsmauern, deren Errichtung bis auf die Höhe der Sohlbankhöhe bzw. bis zum Gewölbeansatz in den Zeitraum zwischen der Mitte des 14. Jahrhunderts und dem 1. Drittel des 15. Jahrhunderts zu setzten ist. Die übrigen Elemente kamen dementgegen erst in der 2. Hälfte des 15. Jahrhunderts zur Ausführung, wobei der langsame Baufortschritt gleich mehrmals die Gelegenheit bot den ursprünglichen Entwurf nach neuen Maßstäben zu modernisieren. Neu ist zudem die Erkenntnis, dass sich das Bauwerk in seinen Anfängen, aber auch in seiner Abschlussphase, als gekonnte Summe zahlreicher gestalterischer Innovationen der mitteleuropäischen Architekturlandschaft präsentiert. Vor diesem Hintergrund wird die Notwendigkeit einer umfassenden Aufarbeitung der erhaltenen Schriftquellen und der bauarchäologischen Bestände besonders deutlich: Denn erst mithilfe dieser könnte der Forschungsstand nachhaltig verbessert und St. Moritz als bedeutendes Denkmal der mitteleuropäischen Architekturlandschaft für die internationale Wissenschaft zugänglich gemacht werden. 


\title{
The Choir of St Maurice in Olomouc in the Interplay of Architectural Innovations of the Late Medieval Central Europe. Old Observations and New Results
}

\author{
Résumé
}

This paper casts new light on the building history of the choir of the parish church St Maurice in Olomouc. By using information taken from written sources relating to the evidence of the church's fabric, it was possible to date the outer walls of the choir to the first phase of the late medieval rebuilding project. Due to the results of a stylistic, typological and archaeological analysis, the edification of this early period of work can be proposed to the second half of the fourteenth century and the first third of the fifteenth century. This thesis revises the traditional dating of the choir between 1453 and 1483/1492 and, thus, places the beginning of the choir into a different political and cultural context. At that time it was the wide-ranging contacts of Olomouc's bishops - due to their expanding estates - that enabled inter-regional connections not only to Bohemia, but also to Silesia. These circumstances made it possible, that the goundplan adopted at this stage - a middle main choir, which is accompanied by side-shortening secondary choirs - was influenced by the older choirs from the same type in Wrocław (St Mary on the Sand, St Elisabeth) and Namysłów (St Peter and Paul). Previous research literature, however, came to another conclusion: the architectures in Steyr (St Egidius and Koloman) and Prague (Church of Our Lady before Týn) was regarded as archetypes of this building. Evidence suggests that the masons incorpo- rated new and innovative designs in the groundplan, as well as in the details: Important paralles found in some church architectures in todays Austria.

In addition to the re-dating of the outer walls of the choir, this paper also reaches a different conclusion in terms for the final decades of the rebuilding work (c. 1450-1520). Following a construction break in the years between 1412/35 and 1453 (during that time, work was done at the nave and the northwestern tower), attention turned back to the sanctuary. This resulted in a shift of stylistic orientation: Particularly in the high vaults, as well as in the wide columns, and further, shown in the inventive forms of tracery and portal jambs. The second phase shows a new approach to design. Almost every aspect of the new design represents close relations to solutions that had been developed in previous works on some of the most innovative lodges of the time in Central Europe during that time, for instance Brno and Vienna. It is necessary to enhance that the second phase is not only notable for its regional artistic connections (especially to the innovative architecture of St James in Brno); rather, it also suggests a particular kindship with Viennese lodge projects, such as St Stephan's in Vienna or the parish churches in Steyr, Eggenburg and Znojmo. Therefore, the choir of St Maurice in Olomouc must be recognized as work of international standing. 